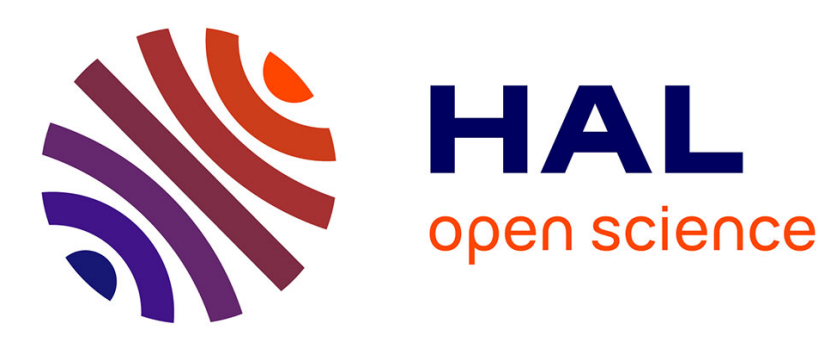

\title{
Phase and gain control policies for robust active vibration control of flexible structures
}

Kai Zhang, Gérard Scorletti, Mohamed Ichchou, F. Mieyeville

\section{To cite this version:}

Kai Zhang, Gérard Scorletti, Mohamed Ichchou, F. Mieyeville. Phase and gain control policies for robust active vibration control of flexible structures. Smart Materials and Structures, 2013, pp.075025. 10.1088/0964-1726/22/7/075025 . hal-00839744

\section{HAL Id: hal-00839744 https://hal.science/hal-00839744}

Submitted on 2 Jan 2014

HAL is a multi-disciplinary open access archive for the deposit and dissemination of scientific research documents, whether they are published or not. The documents may come from teaching and research institutions in France or abroad, or from public or private research centers.
L'archive ouverte pluridisciplinaire HAL, est destinée au dépôt et à la diffusion de documents scientifiques de niveau recherche, publiés ou non, émanant des établissements d'enseignement et de recherche français ou étrangers, des laboratoires publics ou privés. 


\title{
Phase and gain control policies for robust active vibration control of flexible structures
}

\author{
K. Zhang \\ Laboratoire de Tribologie et Dynamique des Systèmes, Ecole Centrale de Lyon, 36 avenue \\ Guy de Collongue, 69134 Ecully Cedex, France \\ G. Scorletti \\ Laboratoire Ampère, Ecole Centrale de Lyon, 36 avenue Guy de Collongue, 69134 Ecully \\ Cedex, France \\ M.N. Ichchou* \\ Laboratoire de Tribologie et Dynamique des Systèmes, Ecole Centrale de Lyon, 36 avenue \\ Guy de Collongue, 69134 Ecully Cedex,France \\ F. Mieyeville \\ L'Institut des Nanotechnologies de Lyon, Ecole Centrale de Lyon, 36 avenue Guy de \\ Collongue, 69134 Ecully Cedex, France
}

\begin{abstract}
The interest of this article is to develop a general and systematic robust control methodology for active vibration control of flexible structures. For this purpose, first phase and gain control policies are proposed to impose qualitative frequency-dependent requirements on the controller to consider a complete set of control objectives. Then the proposed control methodology is developed by employing phase and gain control policies in the dynamic output feedback $H_{\infty}$ control: according to the set of control objectives,
\end{abstract}

\footnotetext{
*Corresponding author

Email address: mohamed.ichchou@ec-lyon.fr (M.N. Ichchou)
} 
phase and gain control policies incorporate necessary weighting functions and determine them in a rational and systematic way; on the other hand, with the appropriate weighting functions efficient $H_{\infty}$ control algorithms can automatically realize phase and gain control policies and generate a satisfactory $H_{\infty}$ controller. The proposed control methodology can be used for both SISO and MIMO systems with collocated or non-collocated sensors and actuators. In this article, it is validated on a non-collocated piezoelectric cantilever beam. Both numerical simulations and experimental results demonstrate the effectiveness of the proposed control methodology.

\section{Keywords}

Phase and gain control policies, $H_{\infty}$ control, non-collocated systems, parametric and dynamic uncertainties

\section{Introduction}

More advanced technologies and materials in industries lead to the implementation of lightweight components for miniaturization and efficiency. Due to the lightweight components, the structures become more flexible and more susceptible to vibrations, which may cause unpleasant noises, unwanted stresses, malfunctions and even failures. As a result, the flexible structures have naturally become suitable candidates for active vibration control and piezoelectric transducers have been widely used for this purpose with various control designs, e.g. PID control (Khot et al., 2011), velocity feedback control (Aoki et al., 2008), positive position feedback control (PPF) (Qiu et al., 2007), acceleration feedback control (AFC) (Qiu et al., 2009), independent modal control (Jemai et al., 1999), linear quadratic regulator (LQR) (Bhat- 
tacharya et al., 2002), fuzzy control (Qu et al., 2004), adaptive control (Ma and Ghasemi-Nejhad, 2005), $\mu$ synthesis (Li et al., 2003) and $H_{\infty}$ control (Iorga et al., 2008).

In spite of the large number of control designs for active vibration control, a general methodology which allows us to systematically design a robust controller that satisfies a complete set of control objectives has to be proposed. The set of control objectives include the vibration reduction of every controlled resonant mode with corresponding a prior determined level, the constraints on the control energy, the reduction of effects of the measurement noise and the stability robustness to parametric and dynamics uncertainties. Besides, as these control objectives usually have conflicting requirements on the controller, the control design must achieve a trade-off among them in a rational and systematic way. In this article, this problem is investigated.

It is demonstrated that, compared to PID, LQR and $H_{2}$ control, the $H_{\infty}$ control can provide better robustness properties in the presence of parametric and dynamic uncertainties (Crassidis et al., 2000; Zhang et al., 2001). It can be applied to both the single-input-single-output (SISO) systems and the multiple-input-multiple-output (MIMO) systems. The $H_{\infty}$ control allows defining the design specifications in the frequency domain. Furthermore, the state and dynamic output feedback $H_{\infty}$ control designs can be accomplished efficiently using polynomial-time algorithms and provide a stabilizing controller with a reasonable order (Doyle et al., 1989; Gahinet and Apkarian, 1994). Due to these features, the $H_{\infty}$ control is receiving intense interest in the control literature and has been successfully applied to a wide variety of practical problems (Jabbari et al., 1995). However despite these promising 
features, practical use of $H_{\infty}$ based active vibration control remains limited mainly due to its drawbacks such as how to incorporate necessary weighting functions and determine them. In the following, we have an extensive review of the $H_{\infty}$ control designs for robust active vibration control:

- The mixed sensitivity design is most usually adopted in $H_{\infty}$ control, e.g. Sadri et al. (1999); Kar et al. (2000a); Seto and Kar (2000); Liu et al. (2004); Douat et al. (2011). However, this $H_{\infty}$ control structure may necessarily lead to the pole-zero cancellation between the designed $H_{\infty}$ controller and the plant. This pole-zero cancellation should be avoided for lightly damped flexible structures, especially in the presence of parametric uncertainties (Sefton and Glover, 1990; Scorletti and Fromion, 2009).

- The definition of the specification of vibration reduction is critical in $H_{\infty}$ control. A frequency-dependent weighting function $W(s)$ or a matching model $M(s)$ can be used to this end (Forrai et al., 2001; Rao et al., 2007). However, it is not explained clearly how to choose $W(s)$ or $M(s)$ and if several resonant modes have to be controlled, $W(s)$ and $M(s)$ could be very complicated and have a high order. This results in a high-order $H_{\infty}$ controller, which requires extensive online computations imposing limitations on the sample rate for real-time implementation and precluding observation and control of high frequency resonant modes.

In addition to the vibration reduction performance, the $H_{\infty}$ control should also impose constraints on the control energy and reduce the 
effects of the measurement noise. But these control objectives are often neglected, e.g. Seto and Kar (2000); Kar et al. (2000b); Forrai et al. (2001); Xie et al. (2004); Liu et al. (2004). Sometimes, constant weighting functions are used for this purpose, e.g. Zhang et al. (2001); Huo et al. (2008). However, as they are frequency-independent and cannot represent suitable requirements on the controller over various frequency ranges, the measurement noise may have significant adverse effects on the control performances and the closed-loop system may even not work properly in real-time implementation due to the control saturation problem.

- To consider a set of control objectives, necessary weighting functions have to be incorporated in $H_{\infty}$ control and determined appropriately. The selection of weighting functions is critical in $H_{\infty}$ control and even regarded to be the main drawback of $H_{\infty}$ control by Zhang et al. (2001). As claimed in Crassidis et al. (2000), the selection of weighting functions cannot be explicitly related to the control objectives in a straightforward manner and trial and error iterations are required to determine the weighting functions. Inappropriate weighting functions may neglect some control objectives and fail to have a satisfactory $H_{\infty}$ controller.

- To consider the stability robustness to parametric and dynamic uncertainties, a norm bounded additive or multiplicative perturbation is widely used in $H_{\infty}$ control. They can represent neglected high frequency dynamics related to the spillover instability, e.g. Yaman et al. 
(2002); Xie et al. (2004); Kar et al. (2000b); Font et al. (1994); Carrere et al. (1997); Caracciolo et al. (2005), and also include all possible uncertain models due to parametric uncertainties, e.g. Crassidis et al. (2000); Forrai et al. (2001); Chang et al. (2002); Filardi et al. (2003); Xie et al. (2004). Based on the unstructured uncertainty, the small gain theorem (Desoer and Vidyasagar, 1975) is then applied to ensure the closed-loop stability.

It is notable that, due to the presence of parametric uncertainties, the employed unstructured uncertainty inevitably introduces considerable conservatism (Morris et al., 1992). To reduce this conservatism, mixed $H_{2} / H_{\infty}$ control together with pole placement is used to guarantee the stability robustness to parametric uncertainties (Hong et al., 2006). But the regulated variables in $H_{2} / H_{\infty}$ control are not clearly specified and there may exist considerable conservatism in the multi-objective state feedback synthesis. Assuming matched form of parametric uncertainties, the singular value decomposition is proposed to consider parametric uncertainties such that the phase margin keeps larger than $60^{\circ}$ for all possible models (Wang et al., 2001; Wang, 2003). However, the matching condition could be violated (Stalford, 1987) and the desired phase or gain margin is no longer guaranteed when the Kalman filter is used for the state estimation (Doyle, 1978). Sometimes, only a dynamic uncertainty is explicitly considered in $H_{\infty}$ control and parametric uncertainties are considered with the $\mu$ analysis to verify the robustness properties with the designed controller, e.g. Yaman et al. (2001, 2002); Iorga et al. (2009). Collocated sensors and actuators are 
also used in $H_{\infty}$ to have prominent stability robustness, e.g. Bai and Grigoriadis (2005); Demetriou et al. (2009). In few cases, neither dynamic nor parametric uncertainty is explicitly considered, e.g. Filardi et al. (2003); Chen et al. (2010).

Besides the $H_{\infty}$ control designs, to reduce the conservatism in the presence of parametric uncertainties or several dynamic uncertainties, Doyle (1982) proposed the concept of structured singular value $(\mu)$ and employed the structured uncertainty $\underline{\Delta}$ to investigate structural characteristics of all uncertainties. Based on $\underline{\boldsymbol{\Delta}}, \mu$ synthesis is developed to design a robust stabling controller such that the robustness properties of the closed-loop system are ensured with respect to the defined $\underline{\Delta}$ (Doyle, 1985; Fan et al., 1991). The motivation of $\mu$ synthesis is attractive, unfortunately, there is no direct method to synthesize such $\mu$ robust controllers. Normally, $\mu$ synthesis involves the use of $H_{\infty}$ optimization for the controller synthesis and $\mu$ analysis for the robustness properties verification with the designed controller, for instance, the widely used $D K$-iteration (Doyle et al., 1991). But even for a given controller, the accurate $\mu$ computation is in general NP-hard ${ }^{1}$ (Braatz et al., 1994; Blondel and Tsitsiklis, 2000). Therefore, lower and upper bounds of $\mu$ are usually calculated to approximate its accurate value with frequency gridding method (Young and Dolye, 1990; Young et al., 1992). This method requires a sufficiently fine frequency gridding to have reliable results. In the case of lightly damped flexible structures, the critical frequency could be ne-

\footnotetext{
${ }^{1}$ given any algorithm to compute $\mu$, there will be problems for which the algorithm cannot find the answer in polynomial time.
} 
glected and the robustness properties are thus overestimated (Freudenberg and Morton, 1992). In addition to the problem introduced by $\mu$ analysis, $D K$-iteration fails to generate a $\mu$ upper bound optimal controller due to its inherent non-convexity and only provides a $\mu$ upper bound sub-optimal controller, which largely depends on the selection of initial parameters. The order of this controller increases in every $D K$-iteration and tends to be very large. The $\mu$ synthesis is often difficult to be formulate directly (Skogestad and Postlethwaite, 2005). As a result, from a practical point of view, $\mu$ synthesis ( $D K$-iteration) is not suitable for active vibration control of flexible strictures.

To avoid the drawbacks of usual $H_{\infty}$ control and $\mu$ synthesis for robust active vibration control of flexible structures, a general and systematic robust control methodology is developed in this article. For this purpose, a positive frequency-dependent function is used to determine the controlled resonant frequencies and define the specification of vibration reduction. To consider the complete set of control objectives, phase and gain control policies are proposed to impose qualitative frequency-dependent requirements on the controller:

- When the specification of vibration reduction is not satisfied, the phase control policy requires the gain of the controller large enough for effective vibration reduction. Meanwhile, it enforces the phase requirement on the controller such that around the controlled resonant frequencies the open-loop transfer function stays in the right half plane on Nyquist plot. This phase property provides adequate stability robustness to parametric uncertainties. The phase requirement is in contrast with 
the passivity theorem (Khalil, 1996) and the negative-imaginary approach (Lanzon and Petersen, 2008) which impose more strict phase requirements on the plant and the controller and can only be applied to collocated systems.

- When the specification of vibration reduction is satisfied, the gain control policy requires the gain of the controller as small as possible to limit the control energy and reduce the effects of the measurement noise. Based on the small gain theorem, it also provides a certain level of stability robustness to a generalized dynamic uncertainty including neglected high frequency dynamics and other dynamics when the phase control policy is not used. As no parametric uncertainty is considered with the small gain terrorem, the associated conservatism is reduced.

Phase and gain control policies are then used in the dynamic output feedback $H_{\infty}$ control to incorporate necessary weighting functions and determine them in a rational and systematic way. On the other hand, with the appropriate weighting functions efficient $H_{\infty}$ control algorithms can automatically realize phase and gain control policies and generate a satisfactory $H_{\infty}$ controller to make a trade-off among various control objectives. The proposed control methodology is developed by well employing phase and gain control policies in the $H_{\infty}$ control. It can be used for both SISO and MIMO systems with collocated or non-collocated sensors and actuators.

The remaining sections of this article are arranged as follows. In Section 2, phase and gain control policies are proposed to consider a complete set of control objectives. In Section 3, phase and gain control policies are used to explain classical AFC and employed in the dynamic output feedback $H_{\infty}$ 
control to develop a general and systematic robust control methodology. In Section 4, the proposed control methodology is validated on a non-collocated piezoelectric cantilever beam with numerical simulations and experimental results. Conclusions are summarized in Section 5.

\section{Controller design}

\subsection{Problem statement}

One of the most significant characteristics of flexible structures is their highly resonant modes due to inherently small dissipation of kinetic and strain energy, which is reflected by a relatively small structural damping. Such flexible structures may experience considerable vibrations when they are excited around the resonant frequencies. To obtain effective vibration reduction, it is desirable to design a controller for the resonance reduction, that is, the controller should effectively reduce the frequency response magnitudes around the controlled resonant frequencies and have limited effects elsewhere.

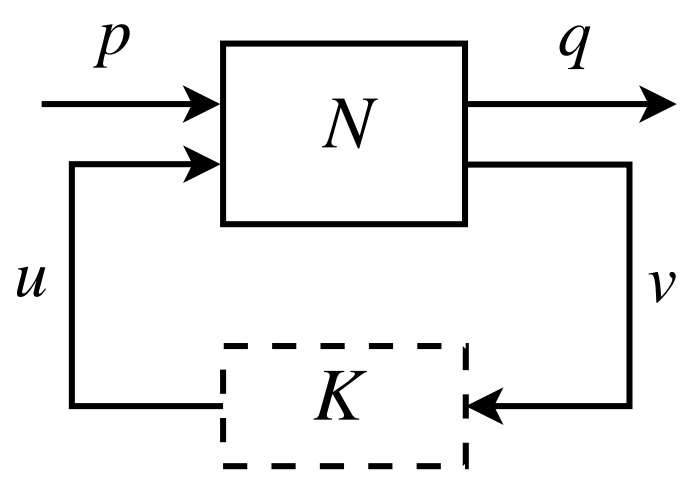

Figure 1: The most general feedback control structure 
To determine the controlled resonant frequencies and quantitatively define the specification of vibration reduction, a positive frequency-dependent function $U(\omega)$ and the most general feedback control structure of Figure 1 are introduced, where $N(s)$ is the general plant, $K(s)$ the stable controller to be designed, $u$ the control signal, $v$ the input signal to $K(s), p=[d, n]^{\prime}$ the external signals including the disturbance signal $d$ and the measurement noise $n$, and $q=[y, u]^{\prime}$ the regulated signals to be minimized including the system output $y$ and the control energy $u$. By partitioning $N(s)$ according to the size of signals, the system is described as

$$
\begin{aligned}
{\left[\begin{array}{l}
q(s) \\
v(s)
\end{array}\right] } & =N(s)\left[\begin{array}{l}
p(s) \\
u(s)
\end{array}\right]=\left[\begin{array}{ll}
N_{q p}(s) & N_{q u}(s) \\
N_{v p}(s) & N_{v u}(s)
\end{array}\right]\left[\begin{array}{l}
p(s) \\
u(s)
\end{array}\right] \\
u(s) & =K(s) v(s)
\end{aligned}
$$

where $N_{q p}(s)$ represents the open-loop transfer function matrix from $p$ to q. The closed-loop transfer function matrix from $p$ to $q$ is given by the linear fractional transformation: $q=F_{l}(N, K)(s) p$, where $F_{l}(N, K)(s)=$ $N_{q p}(s)+N_{q u}(s) K(s)\left(I-N_{v u}(s) K(s)\right)^{-1} N_{v p}(s)$. Denote $T(s)=F_{l}(N, K)(s)$, the closed-loop transfer function from $d$ to $y$ is represented by $T_{y d}(s)$ and the specification of vibration reduction can be defined as

$$
\left|T_{y d}(j \omega)\right| \leq U(\omega), \forall \omega \in \mathbb{R}
$$

where $\mathbb{R}$ denotes the fields of real numbers. For the SISO systems, this specification can be illustrated in Figure 2, where the solid curve is the openloop transfer function from $d$ to $y, N_{y d}(j \omega)$. Obviously, for this particular case, the first two resonant frequencies are the controlled ones. 


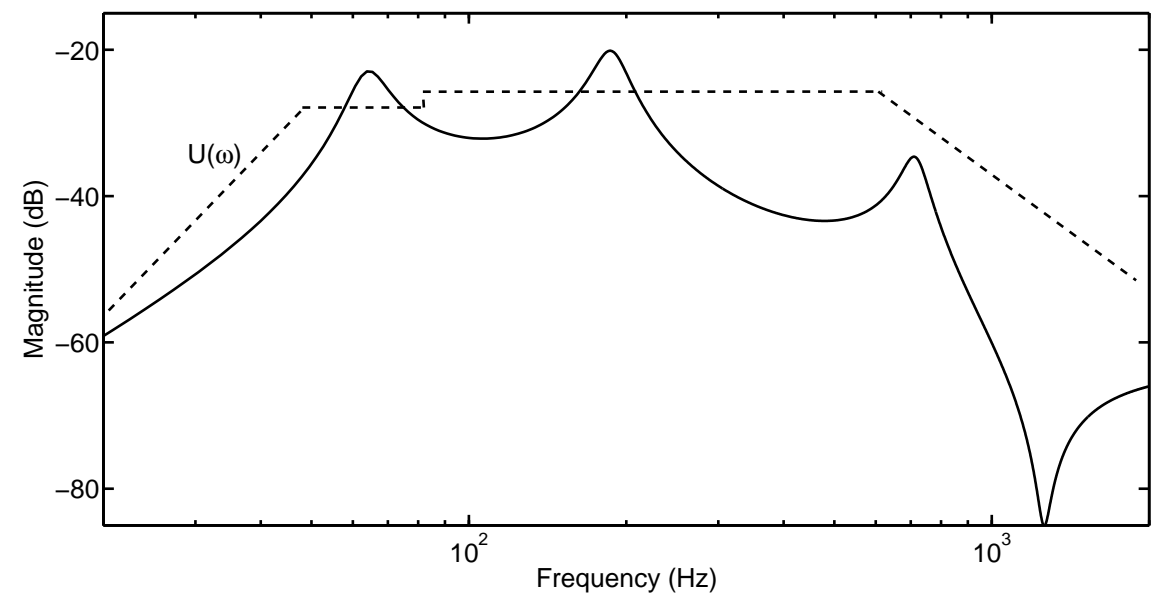

Figure 2: A specification of vibration reduction for flexible structures

In practice, in addition to the specification of vibration reduction, several other control objectives have to be considered simultaneously, e.g. the closedloop stability, the moderate control energy, the effects of the measurement noise and the stability robustness to parametric and dynamic uncertainties. In the control design, the complete set of control objectives can be translated into the requirements on corresponding transfer function matrices. The typical vibration control structure of Figure 3 is introduced for the SISO systems, where $G_{d}(s)$ and $G_{p}(s)$ represent disturbance and plant dynamical models respectively (Pota et al., 1999). This is a specific case of the most general control structure in that the regulated system output $y$ is measured and directly fed back to $K(s)$.

Based on the control structure of Figure 3, the closed-loop stability can be investigated with the Nyquist stability criterion in terms of the openloop transfer function $L(j \omega)=K(j \omega) G_{p}(j \omega)$. The modulus margin $M_{m}$ represents the smallest distance from $L(j \omega)$ to the critical point $-1+j 0$ on 
Nyquist plot (Bourlès and Kwan, 2010),

$$
M_{m}=\inf _{\omega}|1+L(j \omega)|=\frac{1}{\sup _{\omega} \frac{1}{|1+L(j \omega)|}}=\frac{1}{\sup _{\omega}|S(j \omega)|}, \forall \omega \in \mathbb{R}
$$

where $S(j \omega)=(1+L(j \omega))^{-1}$ is the sensitivity function of the closed-loop system. Based on the Nyquist stability criterion, for the stability robustness, the larger $M_{m}$, the better. In addition, $M_{m}^{-1}$ is the maximum peak of the sensitivity function and is closely related to the gain and phase margins (Skogestad and Postlethwaite, 2005). The beneficial effects of $K(s)$ on the vibration reduction is represented by $\left|T_{y d}(j \omega)\right|=\left|G_{d}(j \omega) S(j \omega)\right|$ and the associated control energy is represented by $\left|T_{u d}(j \omega)\right|=\left|G_{d}(j \omega) K(j \omega) S(j \omega)\right|$. The effects of the measurement noise on the control energy and the system output are respectively represented by $\left|T_{u n}(j \omega)\right|=|K(j \omega) S(j \omega)|$ and $\left|T_{y n}(j \omega)\right|=|1-S(j \omega)|=|T(j \omega)|$ termed as the complimentary sensitivity function. Hence, these control objectives are equivalent to reducing the magnitudes of related closed-loop transfer functions.

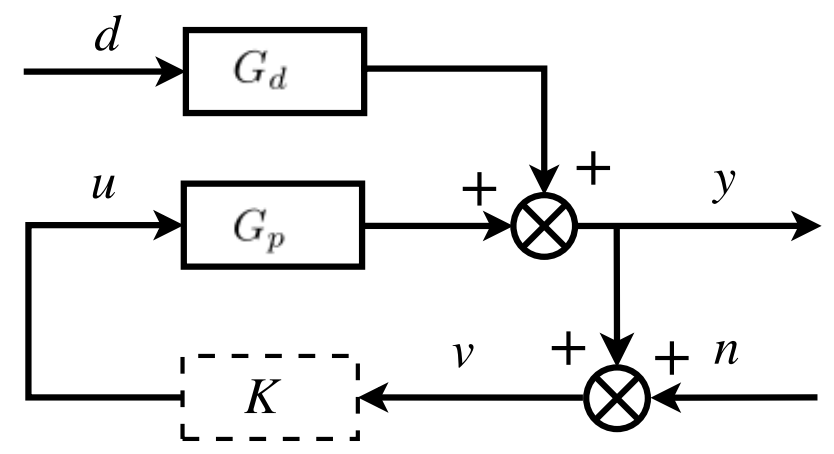

Figure 3: A typical feedback control structure for active vibration control 


\subsection{Phase and gain control policies}

To design a controller $K(j \omega)$ satisfying above mentioned control objectives, it is desirable to translate the control objectives into frequencydependent requirements on $K(j \omega)$. The relationships between the control objectives and the closed-loop transfer functions are used to this end, especially, when $|L(j \omega)| \gg 1$ and $|L(j \omega)| \ll 1$, these closed-loop transfer functions can be simplified with respect to $K(j \omega)$ as summarized in Table 1 . This simplification allows the investigation of the relationships between the control objectives and $|K(j \omega)|$.

\begin{tabular}{|c||c|c|}
\hline$|L(j \omega)|$ & $\gg 1$ & $\ll 1$ \\
\hline \hline$\left|T_{y d}(j \omega)\right|$ & $\approx\left|\frac{G_{d}(j \omega)}{L(j \omega)}\right|$ & $\approx\left|G_{d}(j \omega)\right|$ \\
\hline$\left|T_{y n}(j \omega)\right|$ & $\approx 1$ & $\approx|L(j \omega)|$ \\
\hline$\left|T_{u d}(j \omega)\right|$ & $\approx\left|\frac{G_{d}(j \omega)}{G_{p}(j \omega)}\right|$ & $\approx\left|K(j \omega) G_{d}(j \omega)\right|$ \\
\hline$\left|T_{u n}(j \omega)\right|$ & $\approx\left|\frac{1}{G_{p}(j \omega)}\right|$ & $\approx|K(j \omega)|$ \\
\hline
\end{tabular}

Table 1: Relationships between closed-loop transfer functions and the controller

For efficient vibration reduction, $\left|T_{y d}(j \omega)\right|$ is focused and Table 1 implies that at frequencies where $\left|G_{d}(j \omega)\right|>U(\omega)$, i.e. the specification of vibration reduction is not satisfied, $|K(j \omega)|$ is required to be large enough, for example,

$$
|L(j \omega)| \gg 1 \text { and }|K(j \omega)| \geq \frac{\left|G_{d}(j \omega)\right|}{\left|G_{p}(j \omega) U(\omega)\right|}
$$

On the other hand, at frequencies where $\left|G_{d}(j \omega)\right| \leq U(\omega)$, i.e. the specification of vibration reduction is satisfied, no control energy is needed and the ideal controller should be $|K(j \omega)|=0$. For moderate control energy, $\left|T_{u d}(j \omega)\right|$ has to be limited, however, when $|L(j \omega)| \gg 1$ the control energy is 
nearly independent on $K(j \omega)$ and thus it cannot be limited by any $K(j \omega)$. In contrast, when $|L(j \omega)| \ll 1$ the control energy can be limited by making $|K(j \omega)|$ as small as possible. In addition, when $|L(j \omega)| \ll 1$ the effects of the measurement noise $\left|T_{y n}(j \omega)\right|$ and $\left|T_{u n}(j \omega)\right|$ can also be reduced with small $|K(j \omega)|$. In conclusion, $|K(j \omega)|$ is required to be large enough around the controlled resonant frequencies and beyond these frequencies $|K(j \omega)|$ has to be as small as possible. Above analysis provide available and quite qualitative frequency-dependent requirements on $|K(j \omega)|$. Subsequently, the stability robustness to parametric and dynamic uncertainties is considered and the phase requirement on $K(j \omega)$ is enforced.

\subsubsection{The phase control policy}

The frequency responses of flexible structures are mainly dominated by the behavior around their resonant frequencies. As shown in Figure 4, these frequency responses seem to be circular to some extent on Nyquist plot. The effects of parametric uncertainties on $L(j \omega)$ can also be illustrated on Nyquist plot: when the $i^{\text {th }}$ damping ratio $\zeta_{i}$ is decreasing or the $i^{\text {th }}$ gain $R_{i}$ is increasing, the modulus of the $i^{\text {th }}$ 'circle' becomes larger; when the $i^{\text {th }}$ resonant frequency $\omega_{i}$ is decreasing, the modulus of the $i^{\text {th }}$ 'circle' becomes larger and the orientation of the $i^{\text {th }}$ 'circle' changes. Due to these parametric uncertainties, not only the closed-loop stability but also the stability robustness has to be investigated. Implied by the Nyquist stability criterion, when $L(j \omega)$ is stable and stays in the left half plane (LHP) on Nyquist plot, the effects of parametric uncertainties are critical to the closed-loop stability, particularly, around the controlled resonant frequencies where $|L(j \omega)|$ has to be large enough for effective vibration reduction and 

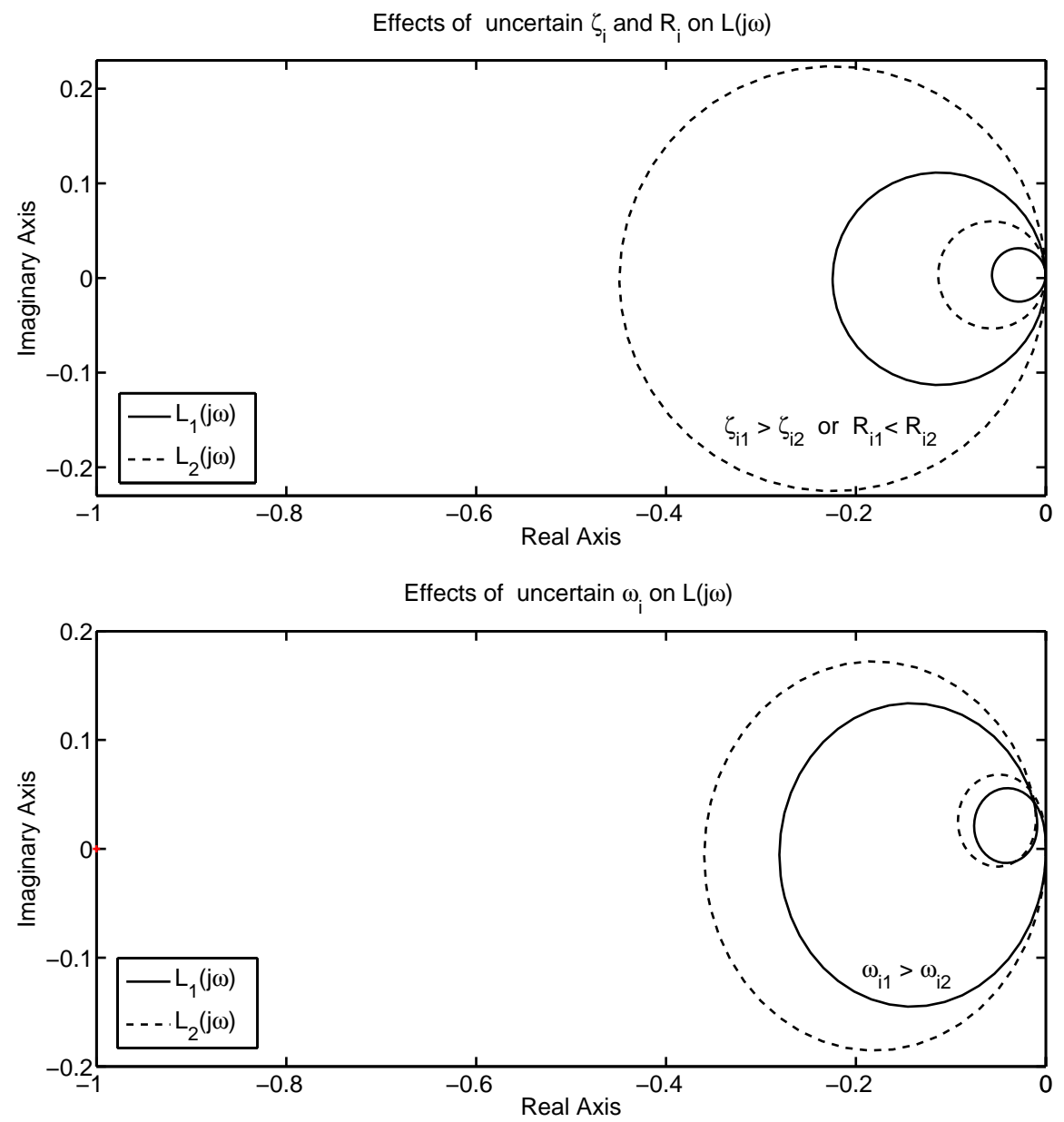

Figure 4: The effects of parametric uncertainties on $L(j \omega)$

thus $L(j \omega)$ may well encircle the critical point $-1+j 0$. To solve this problem, the phase control policy is proposed: around the controlled resonant frequencies, $|K(j \omega)|$ has to be large enough to satisfy the specification of vibration reduction, meanwhile, the stability robustness to parametric uncertainties is guaranteed by enforcing the phase requirement on $K(j \omega)$ such that $\angle L(j \omega)=\left[\angle K(j \omega)+\angle G_{p}(j \omega)\right] \in\left[-90^{\circ},+90^{\circ}\right]$, that is, around the controlled resonant frequencies $L(j \omega)$ stays in the right half plane (RHP) on 
Nyquist plot,

$$
\Re(L(j \omega)) \geq 0, \omega \in\left[\omega_{c i}-\delta_{\omega_{c i}}, \omega_{c i}+\delta_{\omega_{c i}}\right], \delta_{\omega_{c i}}>0
$$

where $\Re(L(j \omega))$ represents the real part of $L(j \omega)$ and $\omega_{c i}$ is the $i^{t h}$ controlled resonant frequency. The Equation (6) guarantees that $L(j \omega)$ cannot intersect the negative real axis on Nyquist plot around $\omega_{c i}$ even there exist a certain level of parametric uncertainties. Necessarily, $L(j \omega)$ cannot encircle the critical point $-1+j 0$ around $\omega_{c i}$ and thus adequate stability robustness to parametric uncertainties is achieved. This phase requirement on $L(j \omega)$ can be regarded as a generalization of the direct velocity feedback control (Balas, 1979), which requires $L(j \omega)$ to stay in RHP at any frequency,

$$
\Re(L(j \omega)) \geq 0, \forall \omega \in \mathbb{R} .
$$

For the SISO systems, the passivity theorem (Khalil, 1996) and the negative-imaginary (NI) approach (Lanzon and Petersen, 2008) can also be interpreted by the phase requirement on $L(j \omega)$. Compared to the phase control policy, more strict phase requirements on $G_{p}(j \omega)$ and $K(j \omega)$ are enforced by these methods to guarantee the closed-loop stability, for example,

$$
\begin{aligned}
& \Re\left(G_{p}(j \omega)\right) \geq 0 \text { and } \Re(K(j \omega))>0, \forall \omega \in \mathbb{R}, \text { the passivity theorem } \\
& \Im\left(G_{p}(j \omega)\right) \leq 0 \text { and } \Im(K(j \omega))<0, \forall \omega \in \mathbb{R}, \text { the NI approach }
\end{aligned}
$$

where $\Im\left(G_{p}(j \omega)\right)$ represents the imaginary part of $G_{p}(j \omega)$. From a practical point of view, it is not necessary and difficult to satisfy these phase requirements for all frequencies, for instance, $\Re\left(G_{p}(j \omega)\right) \geq 0, \forall \omega \in \mathbb{R}$ or $\Im\left(G_{p}(j \omega)\right) \leq 0, \forall \omega \in \mathbb{R}$ can be frequently destroyed by neglected high frequency dynamics or time delays (Rohrs et al., 1985; Griggs et al., 2007). 
Furthermore, these phase requirements cannot be satisfied in the case of non-collocated sensors and actuators, which is often unavoidable due to installation convenience or is even recommendable for high degrees of observability and controllability (Bayon de Noyer and Hanagud, 1998b; Kim and Oh, 2013). In such case, the passivity theorem and the negative-imaginary approach cannot be used. As a result, the uncertainties and non-collocated systems pose challenging problems for the control designs which are proposed to have unconditional closed-loop stability with these methods, e.g. Balas (1979); Sim and Lee (1993); Pota et al. (2002); Aphale et al. (2007); Bhikkaji et al. (2012); Song et al. (2012). In contrast, the phase control policy has no phase requirement on $G_{p}(j \omega)$ and the phase requirement on $K(j \omega)$ has to be satisfied only around $\omega_{c i}$. This allows the application of the phase control policy to both collocated and non-collocated systems to consider not only the stability robustness to parametric uncertainties and but also the specification of vibration reduction.

\subsubsection{The gain control policy}

As above discussed, when the specification of vibration reduction is satisfied, i.e. $\left|G_{d}(j \omega)\right| \leq U(\omega)$, the ideal case is $|K(j \omega)|=0$. However, this is practically impossible and thus the stability robustness to the dynamic uncertainty on $G_{p}(j \omega)$ has to be investigated. Usually, a norm bounded additive or multiplicative perturbation can be used to represent the dynamic uncertainty,

additive perturbation:

$$
G_{p}(j \omega)=G_{p 0}(j \omega)+\Delta_{a}(j \omega), \quad\left|\Delta_{a}(j \omega)\right| \leq\left|W_{a}(j \omega)\right|, \forall \omega \in \mathbb{R}
$$


multiplicative perturbation:

$$
G_{p}(j \omega)=\left(1+\Delta_{m}(j \omega)\right) G_{p 0}(j \omega),\left|\Delta_{m}(j \omega)\right| \leq\left|W_{m}(j \omega)\right|, \forall \omega \in \mathbb{R}
$$

where $G_{p 0}(j \omega)$ and $G_{p}(j \omega)$ are the nominal and perturbed plant dynamical models; $W_{a}(j \omega)$ and $W_{m}(j \omega)$ are norm bounded transfer functions used as upper bounds on the magnitudes of the additive and multiplicative dynamic uncertainties respectively. From the small gain theorem, the necessary and sufficient conditions for the stability robustness to the additive and multiplicative dynamic uncertainties are

additive perturbation:

$$
\left|T_{u n}(j \omega)\right|=\left|K(j \omega) S_{0}(j \omega)\right|<\frac{1}{\left|W_{a}(j \omega)\right|} \leq \frac{1}{\left|\Delta_{a}(j \omega)\right|}, \forall \omega \in \mathbb{R}
$$

multiplicative perturbation:

$$
\left|T_{y n}(j \omega)\right|=\left|T_{0}(j \omega)\right|<\frac{1}{\left|W_{m}(j \omega)\right|} \leq \frac{1}{\left|\Delta_{m}(j \omega)\right|}, \forall \omega \in \mathbb{R}
$$

where $S_{0}(j \omega)=\left(1+K(j \omega) G_{p 0}(j \omega)\right)^{-1}$ and $T_{0}(j \omega)=K(j \omega) G_{p 0}(j \omega) S_{0}(j \omega)$. The smaller $\left|T_{u n}(j \omega)\right|$ and $\left|T_{y n}(j \omega)\right|$ are, the larger $\left|W_{a}(j \omega)\right|$ and $\left|W_{m}(j \omega)\right|$ can be, that is, the closed-loop system can tolerate a larger dynamic uncertainty. From Table 1, when $|L(j \omega)| \ll 1,\left|T_{u n}(j \omega)\right| \approx|K(j \omega)|$ and $\left|T_{y n}(j \omega)\right| \approx|L(j \omega)|$. Hence, above conditions can be reflected by the requirements on $|K(j \omega)|$,

additive perturbation:

$$
|K(j \omega)|<\frac{1}{\left|W_{a}(j \omega)\right|}, \forall \omega \in \mathbb{R}
$$

multiplicative perturbation:

$$
|K(j \omega)|<\frac{1}{\left|G_{p 0}(j \omega) W_{m}(j \omega)\right|}, \forall \omega \in \mathbb{R}
$$


Based on above analysis, the gain control policy is proposed: at the frequencies where the specification of vibration reduction is satisfied, $|K(j \omega)|$ has to be as small as possible to limit the control energy and reduce the effects of the measurement noise. Based on the small gain theorem, the gain control policy also provides a certain level of stability robustness to a generalized dynamic uncertainty including usual neglected high frequency dynamics and other dynamics when the phase control policy is not used, e.g. the low and middle frequency dynamics in Barrault et al. (2007) and Barrault et al. (2008). In addition, as only the dynamic uncertainty is considered with the small gain theorem, the associated conservatism could be reduced.

The proposed phase and gain control policies impose frequency-dependent requirements on $|K(j \omega)|$ and $\angle K(j \omega)$ to consider a complete set of control objectives in the presence of parametric and dynamic uncertainties. It is notable that phase and gain control policies are quite qualitative, for instance, the $\delta_{\omega_{c i}}$ in Equation (6) is not explicitly specified and related formulation derivations are not rigorous. As it is impossible to change $|K(j \omega)|$ or $\angle K(j \omega)$ dramatically over a very small frequency range, there always exist transition frequency ranges for $K(j \omega)$ to switch from one control policy to the other one. The transition frequency ranges are most critical to control design especially when the resonant modes are closely spaced and the phase control policy has to be used over middle frequency ranges. As a result, to make full use of phase and gain control policies, great attention should be paid to their realization and the trade-off among various control objectives. Although for several specific SISO cases, phase and gain control policies could be realized by some classical control methods such as AFC and so on, it is desirable to 
have a more rational and systematic way to realize them for more general cases. The dynamic output feedback $H_{\infty}$ control is a competitive solution to this problem due to its inherent characteristics.

\section{Application of phase and gain control policies}

\subsection{Explanation of acceleration feedback control}

The basic idea of acceleration feedback control (AFC) is to pass the acceleration signal through some second order compensators with suitable parameters and generate a force feedback proportional to the output of the controller (Bayon de Noyer and Hanagud, 1998b). If $n$ resonant modes of a flexible structure $G(s)$ have to be controlled simultaneously, the AFC controller $K_{A F C}(s)$ has to include $n$ compensators in parallel

$$
\begin{aligned}
G(s) & =\sum_{i=1}^{n} \frac{R_{i} s^{2}}{s^{2}+2 \zeta_{s i} \omega_{s i} s+\omega_{s i}^{2}} \\
K_{A F C}(s) & =\sum_{i=1}^{n} \frac{\gamma_{i} \omega_{c i}^{2}}{s^{2}+2 \zeta_{c i} \omega_{c i} s+\omega_{c i}^{2}}
\end{aligned}
$$

where $\omega_{s i}, \zeta_{s i}$ and $R_{i}$ are the natural frequency, the damping ratio and the gain of $i^{\text {th }}$ controlled resonant mode of the flexible structure; $\omega_{c i}, \zeta_{c i}$ and $\gamma_{i}$ are corresponding parameters of $K_{A F C}(s)$. The principle structure of AFC is shown in Figure 5, where each compensator is just tuned to a controlled resonant mode. This control structure is a specific case of the general control structures since the regulated system output $y$ can be measured and directly fed back to the controller. In addition, the disturbance $d$ and the plant input $u$ are assumed to be exerted at the same position. The structure of $K_{A F C}(s)$ is fixed and the focus of AFC is to determine the parameters of $K_{A F C}(s)$ for every controlled resonant mode. 


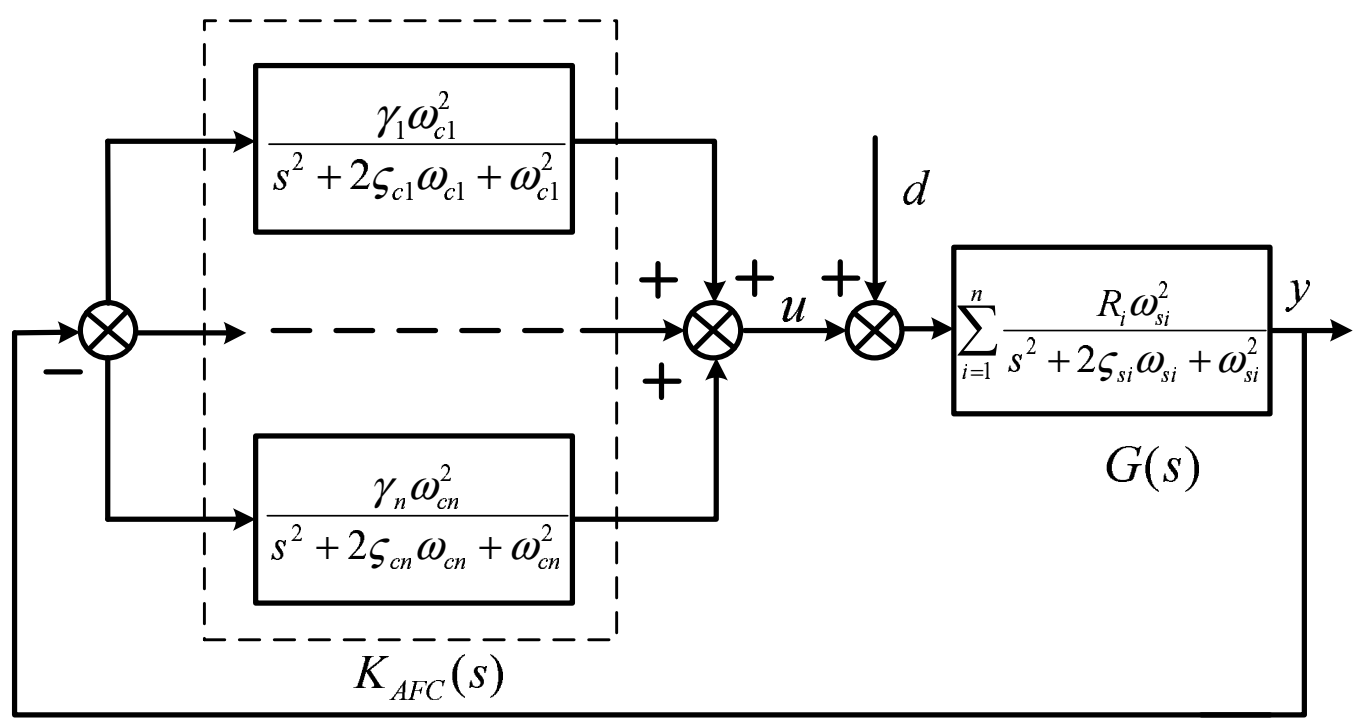

Figure 5: The principle of AFC for $n$ controlled resonant modes

According to the phase control policy, $\omega_{c i} \approx \omega_{s i}$ and appropriate $\zeta_{c i}, \gamma_{i}$ are used to ensure $\left|K_{A F C}(j \omega)\right|$ large enough around $\omega_{s i}$. In this case, $L(j \omega)$ can be approximated as

$$
L(j \omega)=G(j \omega) K_{A F C}(j \omega) \approx \frac{\gamma_{i} R_{i}}{4 \zeta_{c i} \zeta_{s i}}, \omega \in\left[\omega_{s i}-\delta_{\omega_{s i}}, \omega_{s i}+\delta_{\omega_{s i}}\right]
$$

This implies that, around $\omega_{s i}, \gamma_{i} R_{i}>0$ ensures $\Re(L(j \omega))>0$ and $|L(j \omega)|$ is proportional to $\gamma_{i} / \zeta_{c i}$. Therefore, the selection of $\zeta_{c i}$ and $\gamma_{i}$ has significant effects on the vibration reduction performance. Due to the fixed structure of $K_{A F C}(j \omega)$, the gain control policy can only be used after $\omega_{s n}$ where $K_{A F C}(j \omega)$ begins to roll off.

Above design method of $K_{A F C}(s)$ with phase and gain control policies are consistent with the methods in literature, e.g. the critically damped method (Goh and Yan, 1996), the cross-over point method (Bayon de Noyer and Hanagud, 1998b) and the $H_{2}$ optimized method Bayon de Noyer and 
Hanagud (1998a). All of these methods require $\omega_{c i}=\omega_{s i}$ and $\gamma_{i} R_{i}>0$.

\subsection{Proposed control methodology}

As classical control cannot ensure that the designed controllers are optimal with respect to a set of control objectives simultaneously, in this article, a general and systematic robust control methodology is developed by employing phase and gain control policies in the dynamic output feedback $H_{\infty}$ control. As shown in the $H_{\infty}$ control framework of Figure 6, according to the control objectives, the augmented plant $P(s)$ is built by incorporating necessary weighting functions $W_{i}$ into the typical feedback control structure. The weighting functions account for the relative magnitude of signals, their frequency dependence and relative importance. Two exogenous input signals $w=\left[w_{1}, w_{2}\right]^{\prime}$ and three regulated signals $z=\left[z_{1}, z_{2}, z_{3}\right]^{\prime}$ are employed, where $d=W_{d} w_{1}, n=W_{n} w_{2}, z_{1}=W_{y} y, z_{2}=W_{u} u$ and $z_{3}=W_{v} v$. By partitioning $P(s)$ according to the size of signals, the system is described as

$$
\begin{aligned}
{\left[\begin{array}{l}
z(s) \\
v(s)
\end{array}\right] } & =P(s)\left[\begin{array}{l}
w(s) \\
u(s)
\end{array}\right]=\left[\begin{array}{cc}
P_{z w}(s) & P_{z u}(s) \\
P_{v w}(s) & P_{v u}(s)
\end{array}\right]\left[\begin{array}{l}
w(s) \\
u(s)
\end{array}\right] \\
u(s) & =K(s) v(s)
\end{aligned}
$$

where

$$
\begin{array}{ll}
P_{z w}(s)=\left[\begin{array}{cc}
W_{d}(s) G_{d}(s) W_{y}(s) & 0 \\
0 & 0 \\
-W_{d}(s) G_{d}(s) W_{v}(s) & W_{n}(s) W_{v}(s)
\end{array}\right], \quad P_{z u}(s)=\left[\begin{array}{c}
G_{p}(s) W_{y}(s) \\
W_{u}(s) \\
-G_{p}(s) W_{v}(s)
\end{array}\right] \\
P_{v w}(s)=\left[\begin{array}{ll}
-W_{d}(s) G_{d}(s) & \left.W_{n}(s)\right],
\end{array}\right. & P_{v u}(s)=\left[-G_{p}(s)\right]
\end{array}
$$




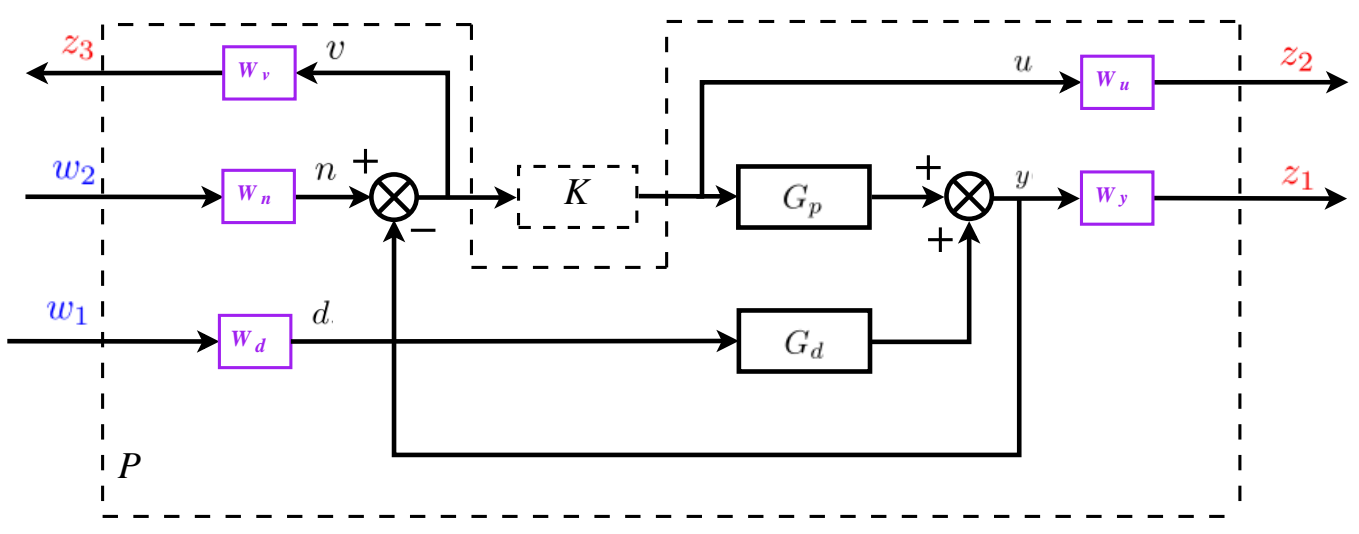

Figure 6: $H_{\infty}$ control framework

The standard $H_{\infty}$ control problem is to achieve a stabilizing controller $K(j \omega)$ which minimizes the $H_{\infty}$ norm of the augmented closed-loop transfer function matrix $F_{l}(P, K)(j \omega)$ defined as

$$
\left\|F_{l}(P, K)(j \omega)\right\|_{\infty}=\max _{\omega} \bar{\sigma}\left(F_{l}(P, K)(j \omega)\right), \forall \omega \in \mathbb{R}
$$

where $F_{l}(P, K)(j \omega)=P_{z w}(j \omega)+P_{z u}(j \omega) K(j \omega)\left(I-P_{v u}(j \omega) K(j \omega)\right)^{-1} P_{v w}(j \omega)$. Let $\gamma_{\text {min }}$ be the minimum value of $\left\|F_{l}(P, K)(j \omega)\right\|_{\infty}$ over all stabilizing controllers. The $H_{\infty}$ sub-optimal control problem is: given a $\gamma>\gamma_{\min }$, find all stabilizing controllers such that $\left\|F_{l}(P, K)(j \omega)\right\|_{\infty} \leq \gamma$. This optimization can be solved efficiently and by reducing $\gamma$ iteratively an optimal solution is achieved (Doyle et al., 1989). With appropriate weighting functions, $\gamma=1$ can be used and a complete set of control objectives are transformed to the constrains on corresponding weighted closed-loop transfer functions, e.g. $\left\|T_{z_{1} w_{1}}(j \omega)\right\|_{\infty} \leq 1$ represents the specification of vibration reduction. Due to the property of $H_{\infty}$ norm, $\left\|F_{l}(P, K)(j \omega)\right\|_{\infty} \leq 1$ ensures $\left\|T_{z_{j} w_{i}}(j \omega)\right\|_{\infty} \leq 1$, that is, these control objectives are satisfied simultaneously with the designed $H_{\infty}$ controller. 
As known, in $H_{\infty}$ control the selection of weighting functions is quite important to achieve a satisfactory $K(j \omega)$. Fortunately, according to a set of control objectives, phase and gain control policies can incorporate necessary weighting functions in $H_{\infty}$ control and determine them in a rational and systematic way:

- To define the specification of vibration reduction, $W_{d}(j \omega)$ and $W_{y}(j \omega)$ should be used and satisfy

$$
\left|W_{d}(j \omega) W_{y}(j \omega) U(\omega)\right| \geq 1, \forall \omega \in \mathbb{R} .
$$

then $\left\|T_{z_{1} w_{1}}(j \omega)\right\|_{\infty}=\left\|W_{d}(j \omega) G_{d}(j \omega) S(j \omega) W_{y}(j \omega)\right\|_{\infty} \leq 1$ ensures $\left|T_{y d}(j \omega)\right|=\left|G_{d}(j \omega) S(j \omega)\right| \leq U(\omega), \forall \omega \in \mathbb{R}$. Depending on the shape of $U(\omega)$, sometimes complicated $W_{d}(j \omega)$ and $W_{y}(j \omega)$ may be required and thus decomposed $H_{\infty}$ control structure is recommendable in such cases.

- To impose the requirements on $K(j \omega)$ according to phase and gain control policies, $|K(j \omega) S(j \omega)|$ can be investigated since it is a good indicator of $|K(j \omega)|$ when $|L(j \omega)| \ll 1$ as shown in Table 1 . When the phase control policy is used, $|K(j \omega)|$ has to be large enough for effective vibration reduction. From the Equation $(5), W_{n}(j \omega)$ and $W_{u}(j \omega)$ should be used and satisfy

$$
\left|W_{n}(j \omega) W_{u}(j \omega) G_{d}(j \omega)\right|<\left|G_{p}(j \omega) U(j \omega)\right|, \forall \omega /\left|G_{d}(j \omega)\right|>U(\omega)
$$

The phase requirement on $K(j \omega)$ can be automatically fulfilled by the $H_{\infty}$ control algorithm with a stable stabilizing $K(j \omega)$. This provides adequate stability robustness to parametric uncertainties. When the 
gain control policy is used, $|K(j \omega)|$ has to be as small as possible to have moderate control energy and reduce the effects of the measurement noise. Besides, the gain control policy has to provide a certain level of stability robustness to a dynamic uncertainty. For this purpose, with the additive dynamic uncertainty $\Delta_{a}(j \omega), W_{n}(j \omega)$ and $W_{u}(j \omega)$ should be used and satisfy

$$
\left|W_{n}(j \omega) W_{u}(j \omega)\right|>\left|W_{a}(j \omega)\right|, \forall \omega \in \mathbb{R}
$$

then $\left\|T_{z_{2} w_{2}}(j \omega)\right\|_{\infty}=\left\|W_{n}(j \omega) K(j \omega) S(j \omega) W_{u}(j \omega)\right\|_{\infty} \leq 1$ ensures the stability robustness to $\Delta_{a}(j \omega)$ based on Equation (9); with the multiplicative dynamic uncertainty $\Delta_{m}(j \omega), W_{n}(j \omega)$ and $W_{y}(j \omega)$ should be used and satisfy

$$
\left|W_{n}(j \omega) W_{y}(j \omega)\right|>\left|W_{m}(j \omega)\right|, \forall \omega \in \mathbb{R}
$$

then $\left\|T_{z_{1} w_{2}}(j \omega)\right\|_{\infty}=\left\|W_{n}(j \omega) T(j \omega) W_{y}(j \omega)\right\|_{\infty} \leq 1$ ensures the stability robustness to $\Delta_{m}(j \omega)$ based on Equation (10).

- To have a modulus margin $M_{m}>\lambda \in(0,1), W_{n}(j \omega)$ and $W_{v}(j \omega)$ should be used and satisfy

$$
\left|W_{n}(j \omega) W_{v}(j \omega)\right|>\lambda, \forall \omega \in \mathbb{R}
$$

This can be derived from Equation (4) and the constrain on $|S(j \omega)|$

$$
\left\|T_{z_{3} w_{2}}(j \omega)\right\|_{\infty}=\left\|W_{n}(j \omega) S(j \omega) W_{v}(j \omega)\right\|_{\infty} \leq 1
$$

For instance $\lambda=0.5$ implies that $\sup |S(j \omega)|$ must be less than 2 and thus it is required $\left|W_{n}(j \omega) W_{v}(j \omega)\right|^{\omega}>0.5, \forall \omega \in \mathbb{R}$. 
As shown above, according to the set of control objectives, phase and gain control policies can be used in $H_{\infty}$ control to incorporate necessary weighting functions and determine them in a rational and systematic way. On the other hand, with the appropriate weighting functions efficient $H_{\infty}$ control algorithms can automatically realize phase and gain control policies and generate a satisfactory $H_{\infty}$ controller to make a trade-off among various control objectives. Although the phase control policy is interpreted with the SISO systems, a nice point is that the $H_{\infty}$ control can be also used for the control design of MIMO systems. As a result, a general and systematic robust control methodology for active vibration control of flexible structures is developed by well employing phase and gain control policies in the dynamic feedback output feedback $H_{\infty}$ control. It can be used for both SISO and MIMO systems with collocated or non-collocated sensors and actuators.

\section{Numerical simulation \& experimental results}

\subsection{System modeling}

To illustrate the effectiveness of the proposed control methodology, active vibration control of a non-collocated piezoelectric cantilever beam is investigated as shown in Figure 7, where a piezoelectric actuator is mounted near the fixed end and an accelerometer near the free end. Based on modal analysis approach (Meirovitch, 1986) and the modeling of piezoelectric actuators (Moheimani and Fleming, 2006), applying Laplace transformation and assuming zero initial conditions, the plant dynamical model $G_{p}(s)$ representing the dynamics from the voltage applied on the piezoelectric actuator 
$V_{a}\left(x_{a}, s\right)$ to the beam acceleration $\ddot{Y}(x, s)$ is

$$
G_{p}(s)=\frac{\ddot{Y}(x, s)}{V_{a}\left(x_{a}, s\right)}=\sum_{i=1}^{\infty} \frac{R_{i} s^{2}}{s^{2}+2 \zeta_{i} \omega_{i} s+\omega_{i}^{2}}
$$

Similarly, the disturbance dynamical model $G_{d}(s)$ representing the dynamics from the disturbance $d\left(x_{d}, s\right)$ to the beam acceleration $\ddot{Y}(x, s)$ is

$$
G_{d}(s)=\frac{\ddot{Y}(x, s)}{d\left(x_{d}, s\right)}=\sum_{j=1}^{\infty} \frac{R_{j} s^{2}}{s^{2}+2 \zeta_{j} \omega_{j} s+\omega_{j}^{2}}
$$

where $R_{i / j}, \zeta_{i / j}, \omega_{i / j}$ are modal parameters to be identified.

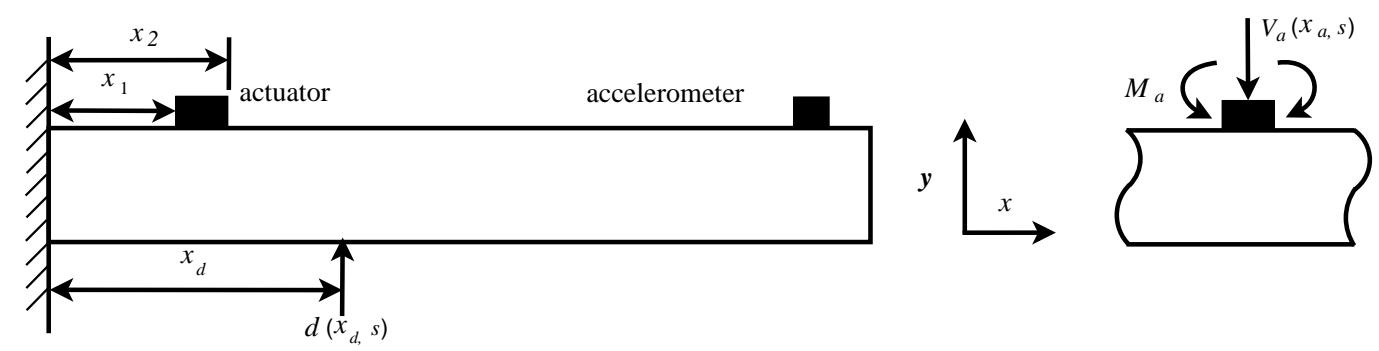

Figure 7: The piezoelectric cantilever beam

The experimental set-up for the parameter identification is illustrated in Figure 8, where the dSPACE generates and acquires the input signal $x(t)$, pseudo random binary sequence (PRBS), and acquire the output signal $y(t)$ from the accelerometer. Experimental frequency responses are estimated by $T_{x y}(\omega)$, being the quotient of the cross power spectral density of $x(t)$ and $y(t), S_{x y}(\omega)$, and the power spectral density of $x(t), S_{x x}(\omega)$ (Bendat and Piersol, 1980),

$$
T_{x y}(\omega)=\frac{S_{x y}(\omega)}{S_{x x}(\omega)}, \omega \in\left\{\omega_{1}, \omega_{2}, \ldots, \omega_{M}\right\}
$$

where $M$ is the number of estimated frequency points. For $G_{p}(s)$, PRBS is sent to the piezoelectric actuator with no input to the shaker. Similarly, 
PRBS is sent to the shaker for $G_{d}(s)$ and the signal to the piezoelectric actuator is set zero. To avoid aliasing problem, the sampling frequency of dSPACE is set at $10 \mathrm{kHz}$. The Hanning window and twenty averages are employed to have reliable experimental frequency responses as shown in Figure 9.

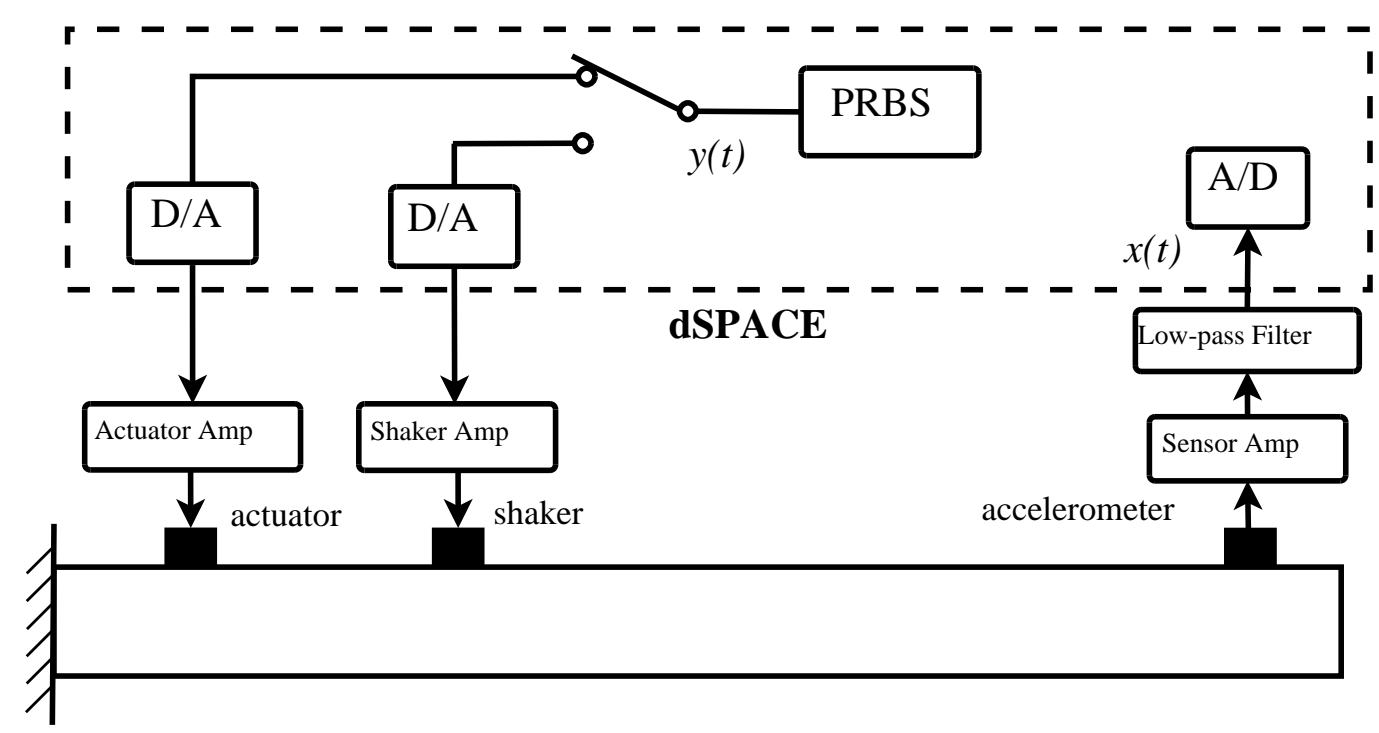

Figure 8: Experimental set-up for parameter identification

With $T_{x y}(\omega), G_{d}(s)$ and $G_{p}(s)$ can be estimated as a ratio of two polynomials in the Laplace variable $s$ based on Equation (23) and (24) with the user-defined number of poles and zeros. The best curve fitting is performed to determine the values of poles, zeros and gains with a least squares method (Schoukens and Pintelon, 1991),

$$
\min _{P} \sum_{k=1}^{M} \varphi(\omega(k))\left|T_{x y}(\omega(k))-G(\omega(k))\right|^{2}, k \in\{1,2, \ldots, M\}
$$

where $P$ represents all the modal parameters of $G(j \omega)$ to identify and $\varphi(\omega(k))$ is a frequency-dependent weighting function to emphasize the importance over corresponding frequencies. Above parameter identification procedure 

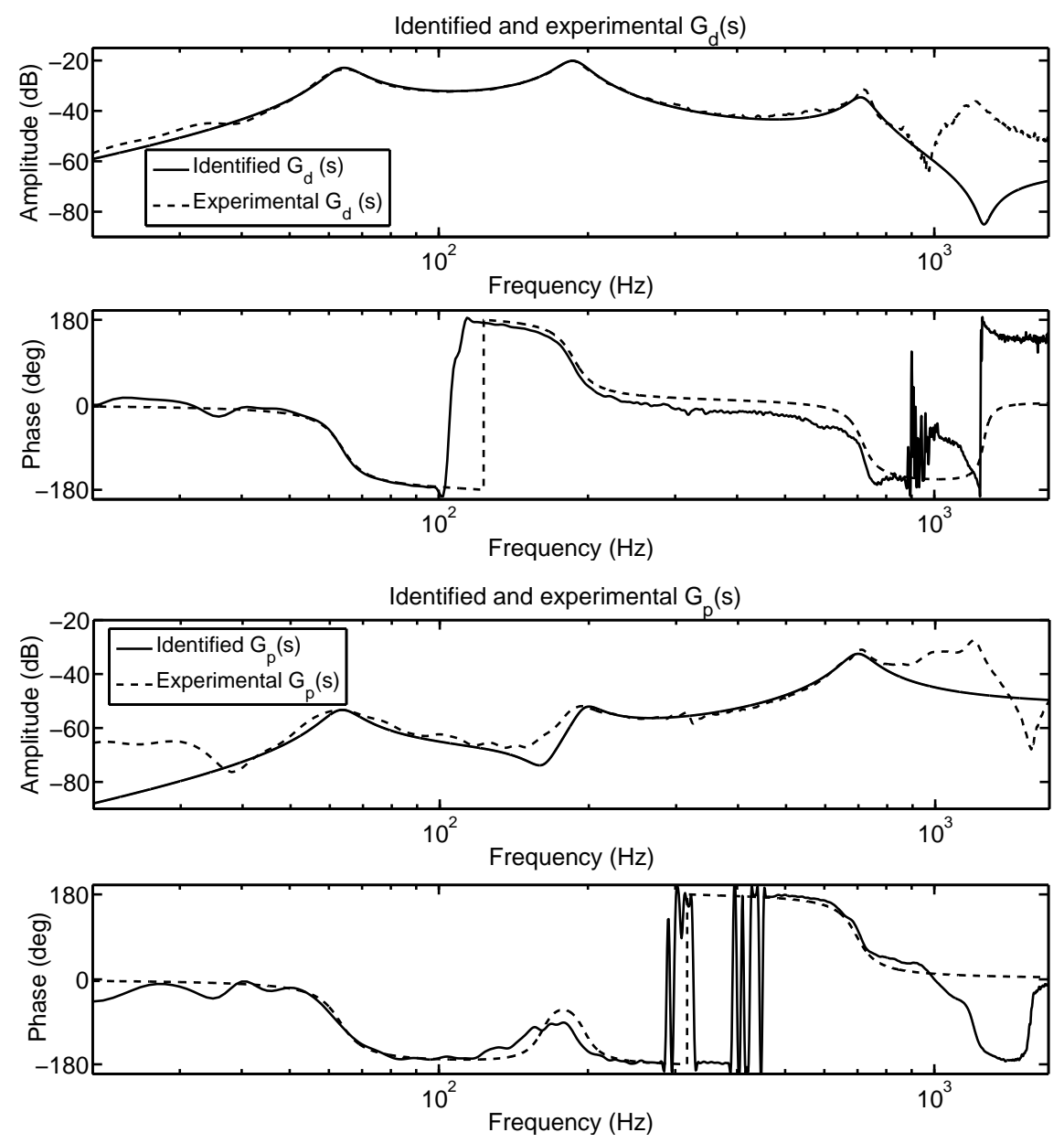

Figure 9: Identified and experimental $G_{d}(s)$ and $G_{p}(s)$

can be realized in Matlab with a graphical user interface. This helps us to obviously observe the contribution of every resonant mode to the whole dynamics. The dynamics of the shaker, the piezoelectric actuator, the accelerometer, the filters and other hardwares are all incorporated into the identified $G_{d}(s)$ and $G_{p}(s)$ :

$$
\begin{aligned}
& G_{d}(s)=\frac{-1.2 \times 10^{-2} s^{2}}{s^{2}+65.8 s+1.6 \times 10^{5}}+\frac{1.4 \times 10^{-2} s^{2}}{s^{2}+172.9 s+1.4 \times 10^{6}}+\frac{-2.1 \times 10^{-3} s^{2}}{s^{2}+505.3 s+2.0 \times 10^{7}} \\
& G_{p}(s)=\frac{-3.6 \times 10^{-4} s^{2}}{s^{2}+65.6 s+1.6 \times 10^{5}}+\frac{-2.8 \times 10^{-4} s^{2}}{s^{2}+153.0 s+1.5 \times 10^{6}}+\frac{3.3 \times 10^{-3} s^{2}}{s^{2}+609.1 s+1.7 \times 10^{7}}
\end{aligned}
$$


As illustrated in Figure 9, the identified frequency responses of $G_{d}(s)$ and $G_{p}(s)$ are in good agreement with the experimental ones over the frequency range of interest. It is notable that either from analytical or finite element method (Moheimani and Fleming, 2006; Piefort, 2001) different transfer functions associated with the same structure should have identical poles, but due to the errors in the system identification, the poles of identified $G_{d}(s)$ and $G_{p}(s)$ are not exactly the same. Based on the specification of vibration reduction as illustrated in Figure 2, the phase control policy has to be applied to the first two resonant modes and the gain control policy to the others.

\subsection{Design of $A F C$}

With the cross-over point method (Bayon de Noyer and Hanagud, 1998b), the parameters of $K_{A F C}(s)$ are determined as $\omega_{c i}=\omega_{s i}, \zeta_{c i}=2 \zeta_{f i}-\zeta_{s i}$ and $\gamma_{i}=\frac{\left(\zeta_{c i}-\zeta_{s i}\right)^{2}}{R_{i}}$. The $\zeta_{f i}$ is a user-defined final damping ratio of the $i^{t h}$ controlled resonant mode and the final frequency $\omega_{f i}=\sqrt{\omega_{s i} \omega_{c i}}=\omega_{s i}$. Based on above identified $G_{p}(s)$, with $\zeta_{f 1}=0.3$ and $\zeta_{f 2}=0.2, K_{A F C 1}(s)$ and $K_{A F C 2}(s)$ are designed for the first resonant mode and the first two respectively,

$$
\begin{aligned}
K_{A F C 1}(s) & =\frac{-8.4 \times 10^{7}}{s^{2}+410.8 s+1.6 \times 10^{5}} \\
K_{A F C 2}(s) & =\frac{-8.4 \times 10^{7}}{s^{2}+410.8 s+1.6 \times 10^{5}}+\frac{-4.1 \times 10^{8}}{s^{2}+831.9 s+1.5 \times 10^{6}}
\end{aligned}
$$

The numerical simulations with $K_{A F C 1}(s), K_{A F C 2}(s)$ and the identified models are illustrated in Figure 10. As required by the phase control policy around the controlled resonant frequencies $\omega_{c i},\left|K_{A F C 1}(j \omega)\right|$ and $\left|K_{A F C 2}(j \omega)\right|$ are large enough for effective vibration control and $L(j \omega)$ stays in RHP to have the stability robustness to parametric uncertainties. On the other hand, as required by the gain control policy, $K_{A F C 1}(j \omega)$ and $K_{A F C 2}(j \omega)$ roll off af- 
Phase and gain control policies with AFC

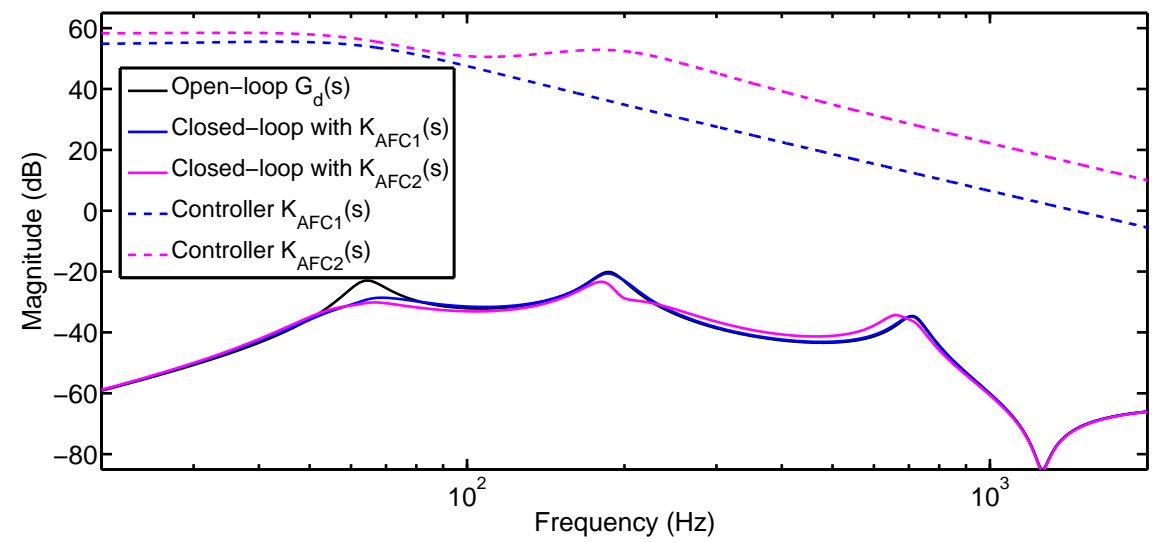

Phase and gain control policies with AFC

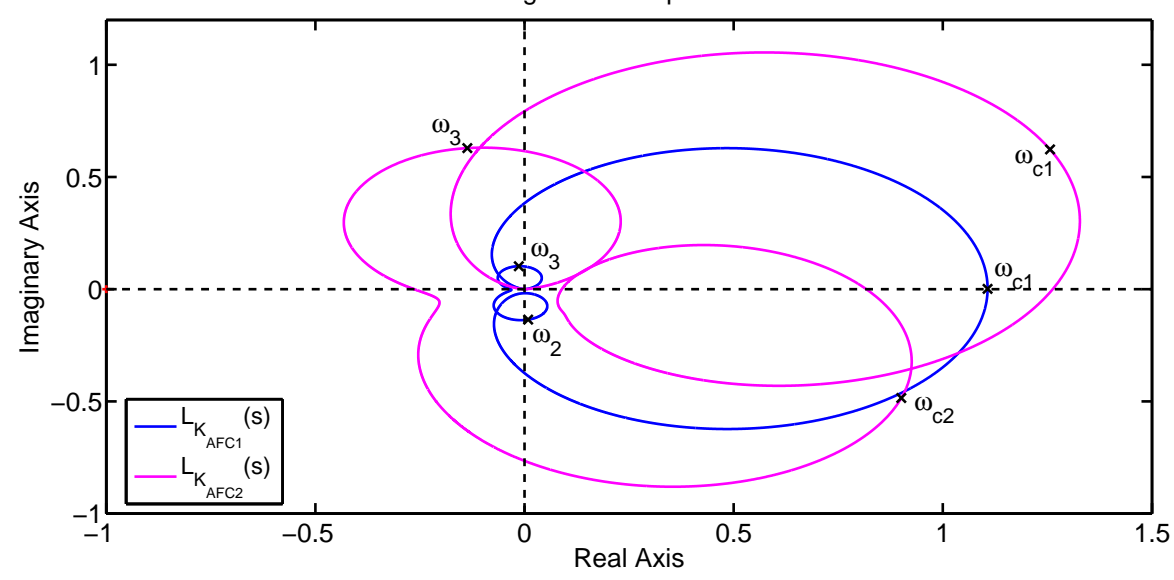

Figure 10: Phase and gain control policies with AFC: $\omega_{c i}$ represents the $i^{\text {th }}$ controlled resonant frequency 
ter $\omega_{c 1}$ and $\omega_{c 2}$ respectively to have a certain level of stability robustness to the dynamic uncertainty.

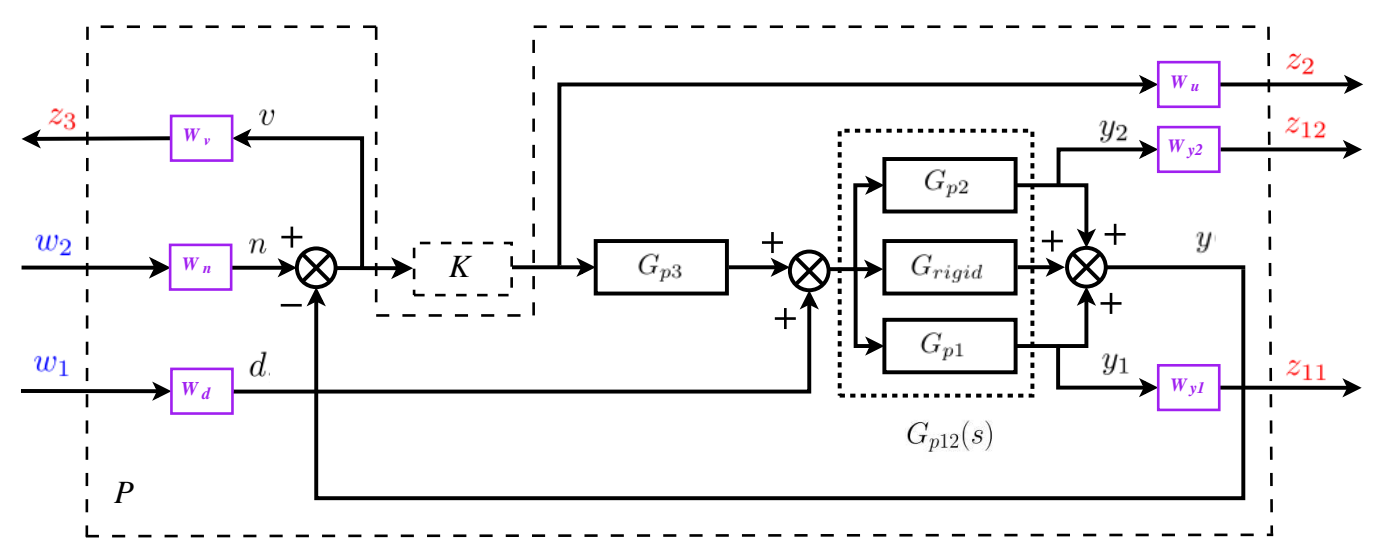

Figure 11: Decomposed $H_{\infty}$ control framework

\subsection{Design of proposed control methodology}

Considering the fact that $G_{d}(s)$ and $G_{p}(s)$ should have the same poles and motivated by the work in Font et al. (1994), for this particular case, $G_{p}(s)$ can be decomposed as $G_{p}(s)=G_{p 12}(s) G_{p 3}(s)$, where $G_{p 12}(s) \approx G_{d 1}(s)+G_{d 2}(s)$. The phase control policy is applied to $G_{p 12}(s)$ and the gain control policy is applied to other dynamics. Moreover, to simplify $W_{d}(j \omega)$ and $W_{y}(j \omega)$ required to reflect the specification of vibration reduction, $G_{p 12}(s)$ is decomposed as illustrated in Figure 11. With this decomposition, constant $W_{d}(j \omega)$, $W_{y 1}(j \omega)$ and $W_{y 2}(j \omega)$ can be used to represent the specification of vibration reduction

$$
\begin{aligned}
& \left\|T_{z_{11} w_{1}}(j \omega)\right\|_{\infty}=\left\|W_{d}(j \omega) G_{p 1}(j \omega) S(j \omega) W_{y 1}(j \omega)\right\|_{\infty} \leq 1 \\
& \left\|T_{z_{12} w_{1}}(j \omega)\right\|_{\infty}=\left\|W_{d}(j \omega) G_{p 2}(j \omega) S(j \omega) W_{y 2}(j \omega)\right\|_{\infty} \leq 1
\end{aligned}
$$


It is notable that $W_{d}(j \omega), W_{y 1}(j \omega)$ and $W_{y 2}(j \omega)$ can also explicitly prevent the pole-zero compensation between $G_{p}(j \omega)$ and $K(j \omega)$ at $\omega_{1}$ and $\omega_{2}$ (Scorletti and Fromion, 2009). These decompositions reduce the order of $H_{\infty}$ controller, being the total order of all involved plants and weighting functions. For the sake of simplicity, $W_{y}(j \omega)$ is no longer used in the decomposed $H_{\infty}$ control framework and thus only the additive dynamic uncertainty is explicitly considered with $W_{n}(j \omega)$ and $W_{u}(j \omega)$.

For this particular case, the proposed control methodology generates the controller $K_{\infty}(s)$ using all constant weighting functions and the popular balanced truncation method ( $\mathrm{Gu}$ et al., 2005) is used to have $K_{r \infty}(s)$ with a reduced order for easier real-time implementation,

$$
\begin{aligned}
K_{\infty}(s) & =\frac{1268.4\left(s-4.3 \times 10^{5}\right)\left(s^{2}-67.8 s+2.5 \times 10^{5}\right)\left(s^{2}+609.1 s+1.9 \times 10^{7}\right)}{\left(s^{2}+408.9 s+3.2 \times 10^{5}\right)\left(s^{2}+950.4 s+9.0 \times 10^{5}\right)\left(s^{2}+4167 s+1.6 \times 10^{7}\right)} \\
K_{r \infty}(s) & =\frac{45134\left(s-1.1 \times 10^{4}\right)\left(s^{2}-70.2 s+2.5 \times 10^{5}\right)}{\left(s^{2}+354.5 s+2.0 \times 10^{5}\right)\left(s^{2}+682 s+8.7 \times 10^{6}\right)}
\end{aligned}
$$

The numerical simulations with $K_{\infty}(s), K_{r \infty}(s)$ and the identified models are illustrated in Figure 10. As required by the phase control policy around $\omega_{c 1}$ and $\omega_{c 2},\left|K_{\infty}(j \omega)\right|$ and $\left|K_{r \infty}(j \omega)\right|$ are large enough for effective vibration control and $L(j \omega)$ stays in RHP to have the stability robustness to parametric uncertainties. On the other hand, as required by the gain control policy, $K_{\infty}(j \omega)$ and $K_{r \infty}(j \omega)$ roll off after $\omega_{c 2}$ to have a certain level of stability robustness to the dynamic uncertainty.

\subsection{Comparisons between $A F C$ and proposed control methodology}

From the numerical simulations, it is shown that for this particular case both AFC and the proposed control methodology achieve the vibration reduction of their controlled resonant modes. However, the specification of vibration reduction is not directly considered by AFC. It is reflected by the 

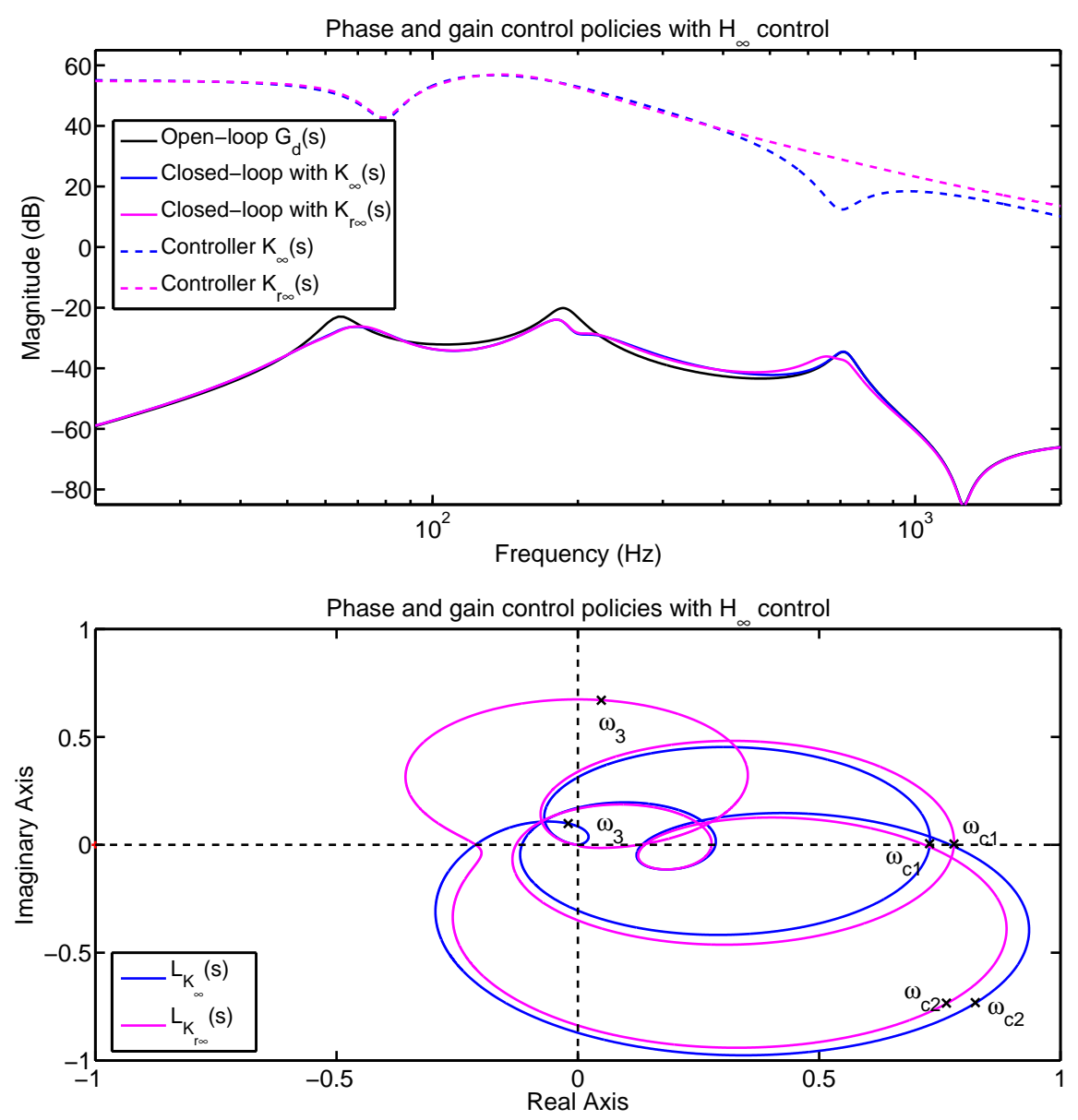

Figure 12: Phase and gain control policies with $H_{\infty}$ control: $\omega_{c i}$ represents the $i^{\text {th }}$ controlled resonant frequency

user-defined damping ratios such as $\zeta_{f 1}=0.3$ and $\zeta_{f 2}=0.2$. The parametric uncertainties could have considerable detrimental effects on the practically obtained damping rations. Besides, when the controlled resonant modes are closely spaced, it is not easy to determine the parameters of $K_{A F C}(j \omega)$ and a large amount of time and energy could be required to meet the specification of vibration reduction. Sometimes, even a lot of efforts are put into 
the parameter selection, no satisfactory $K_{A F C}(j \omega)$ is obtained. This may lead to the question: with respect to the specification of vibration reduction, whether there exists a satisfactory $K_{A F C}(j \omega)$ or not. Fortunately, the proposed control methodology has no such question and a trade-off among various control objectives can be achieved by tuning the weighting functions.

In real-time implementation, due to the physical limitations, it is necessary to enforce a upper bound on $U_{\max }=\max _{t}|u(t)|, \forall t \in \mathbb{R}$ to avoid the controller saturation and exceeding the actuator operated voltage. It is normally difficult to enforce the constraint on $U_{\max }$ directly in $H_{\infty}$ control, however, from a practical point of view, $U_{\max }$ can be limited by restricting $|K(j \omega)|$ in the frequency domain. Due to the fixed structure of $K_{A F C}(j \omega)$, it can only roll off after the last controlled resonant mode even the gain control policy is indeed required at lower frequencies. This means that AFC has little flexibility to make a trade-off between the vibration reduction performance and the control energy. An unnecessarily large $U_{\max }$ may be produced. In contrast, the proposed control methodology can provide more flexibility and explicitly limit $|K(j \omega)|$ with frequency-dependent weighting functions, for instance, the controller $K_{\infty}^{\prime}(j \omega)$ is obtained with a first order low-pass $W_{u}(j \omega)$,

$$
\begin{aligned}
K_{\infty}^{\prime}(s)= & \frac{2.78 \times 10^{5}(s-2431)(s+1)\left(s^{2}-228.2 s+2.8 \times 10^{5}\right)}{(s+963.8)\left(s^{2}+607.4 s+1.23 \times 10^{5}\right)\left(s^{2}+413.6 s+6.23 \times 10^{5}\right)} \times \\
& \frac{\left(s^{2}+609.1 s+1.92 \times 10^{7}\right)}{\left(s^{2}+3280 s+1.91 \times 10^{7}\right)}
\end{aligned}
$$

As shown in Figure 13, compared to $K_{\infty}(j \omega)$ obtained with all constant weighting functions, $\left|K_{\infty}^{\prime}(j \omega)\right| \approx\left|K_{\infty}(j \omega)\right|$ around the controlled resonant frequencies for effective vibration reduction and $\left|K_{\infty}^{\prime}(j \omega)\right| \ll\left|K_{\infty}(j \omega)\right|$ at 
low frequencies. Numerical simulations demonstrate that $K_{\infty}^{\prime}(j \omega)$ produces a smaller $U_{\max }$ than $K_{\infty}(j \omega)$ or $K_{A F C 2}(j \omega)$ does.

Above analysis imply that the proposed control methodology may be not the best choice for some specific SISO cases. Sometimes, other simpler control designs such as AFC can also satisfy the control objectives. But the proposed control methodology is more general and more systematic. It can be used for both SISO and MIMO systems to consider a complete set of control objectives and provide enough flexibility to make a trade-off among them.

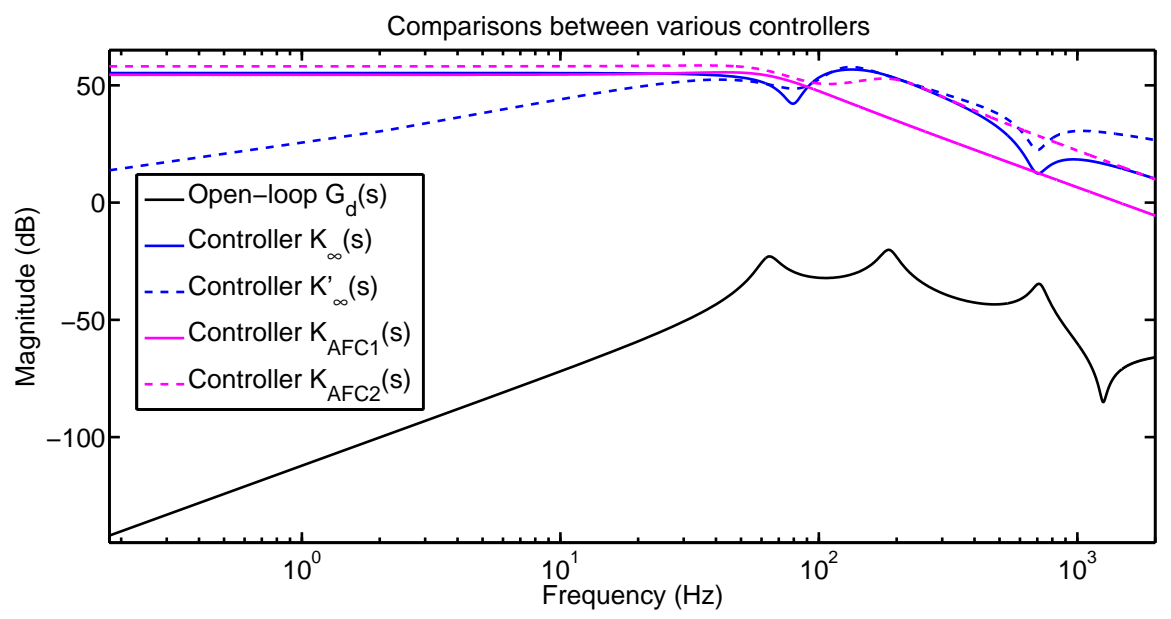

Figure 13: Comparisons between various controllers

\subsection{Experimental implementation}

The experimental set-up for real-time implementation is depicted in Figure 14. The designed continuous controllers are discretized using bilinear transform and compiled to obtain the digital controller codes to upload dSPACE digital control unit with necessary Matlab/Simulink and ControlD- 
esk. The analog-to-digital (A/D) and digital-to-analog (D/A) converters are included in dSPACE hardware. The sampling frequency of dSPACE is set at $10 \mathrm{kHz}$, which is high enough to avoid the aliasing problem. The vibration signal measured by the accelerometer is first through a low-pass filter and then enters the A/D converter. A high-voltage amplifier, capable of driving highly capacitive loads, is used to supply necessary voltage to the piezoelectric actuator. Disturbance signal PRBS with suitable magnitude is generated by dSPACE and sent to a shaker to excite the beam. The offset of the measurement noise is acquired and compensated by adding an external signal with Simulink. It is notable that all amplifies have to keep the same amplification factor as used in the system identification process.

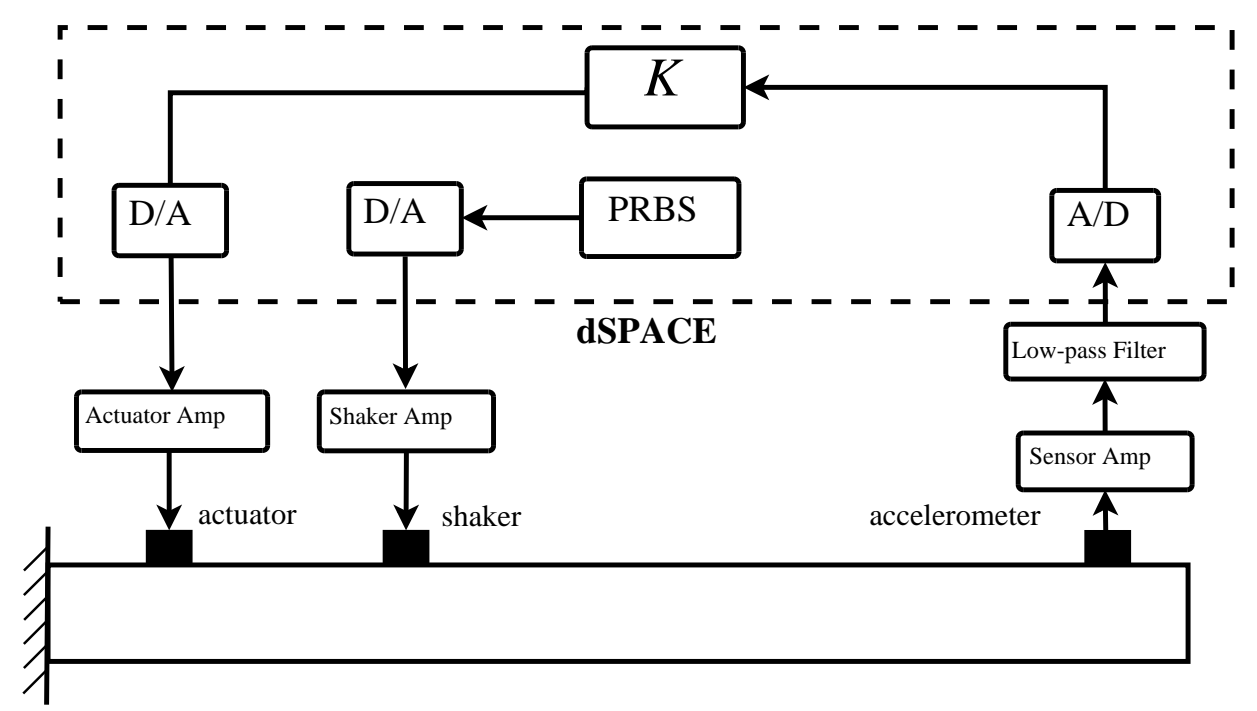

Figure 14: Experimental set-up for active vibration control

Not surprisingly, the output of $\left|K_{A F C 2}(s)\right|$ is saturated. As shown in Figure $15, K_{A F C 1}(s), K_{\infty}(s)$ and $K_{r \infty}(s)$ achieve $8 \mathrm{~dB}$ reduction for the first resonant mode. $K_{\infty}(s)$ and $K_{r \infty}(s)$ also achieve $11 \mathrm{~dB}$ reduction for the sec- 
ond one. The spillover instability due to neglected high frequency dynamics is avoided. Compared to the numerical results calculated with the identified $G_{d}(s)$ and $G_{p}(s)$, the experimental vibration reduction performances are better. To best of our understanding, this performance discrepancy is mainly due to the errors in the system identification, which result in parametric uncertainties on $G_{d}(s)$ and $G_{p}(s), e . g$. the poles of the identified $G_{d}(s)$ and $G_{p}(s)$ are not the same and the realistic $\left|G_{p}(s)\right|$ is indeed larger than the identified one. To have good agreements between numerical and experimental results, more accurate system modeling is desirable. The experimental results also demonstrate that, when the phase control policy is used, the variation in $|L(s)|$ due to parametric uncertainties does not destabilize the system but has considerable effects on the vibration reduction performances. In addition, when the gain control policy is used, $|L(s)|$ should be small enough, otherwise the disturbance signal may be amplified. This problem is most critical over transition frequency ranges, for instance, with $K_{\infty}(s)$ and $K_{r \infty}(s)$ this amplification occurs between the second and third resonant frequencies. As shown in Figure $13,\left|K_{A F C 1}(s)\right| \ll\left|K_{\infty}(s)\right|$ over the transition frequency range and this disturbance amplification is avoided with $K_{A F C 1}(s)$. Therefore, to avoid the disturbance amplification, more accurate system modeling is beneficial and the controller has to roll off quickly enough over the transition frequency ranges. With the proposed control methodology, this roll-off requirement on the controller can be reflected by corresponding weighting functions such as $W_{n}(s)$ and $W_{u}(s)$ of Figure 6 . It is also notable that a trade-off among various control objectives must be considered in the selection of weighting functions. 


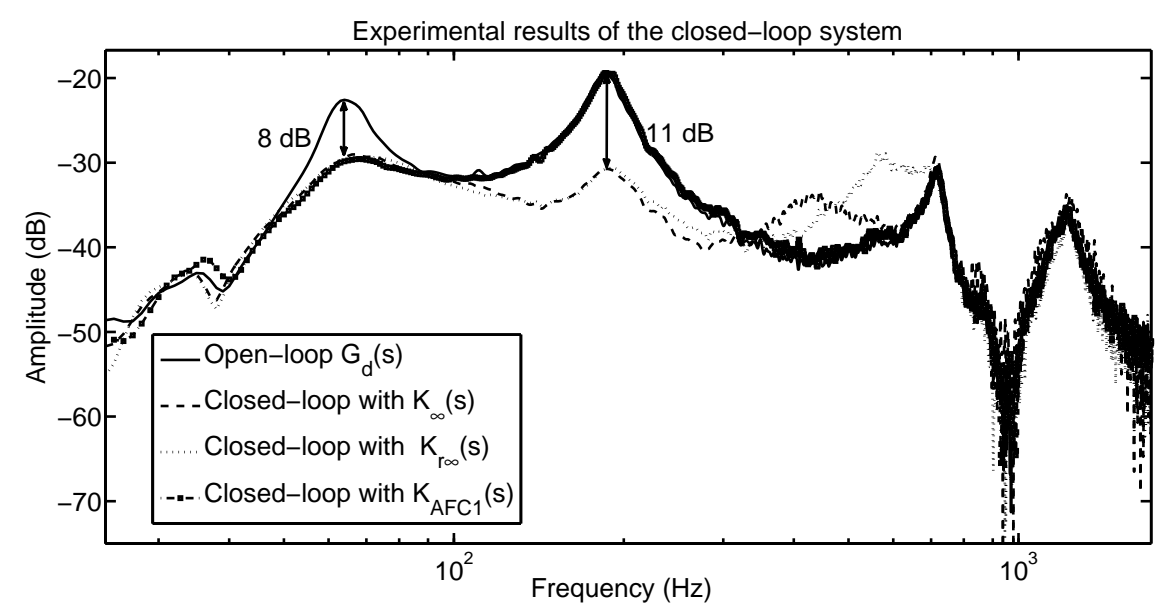

Figure 15: Experimental results of the closed-loop system

\section{Conclusions}

The main contribution of this article is to propose a general and systematic robust control methodology for active vibration control of flexible structures. To achieve this goal, first we have an extensive review of the $H_{\infty}$ based active vibration control of flexible structures, with the focus on the pole-zero cancellation, the considered control objectives, the selection of weighting functions and the stability robustness to parametric and dynamic uncertainties. The limitations of $\mu$ synthesis are also pointed out from a practical point of view. Then phase and gain control policies are proposed to impose qualitative frequency-dependent requirements on the controller to consider a complete set of control objectives. By well employing phase and gain control policies in the dynamic output feedback $H_{\infty}$ control, a general and systematic robust control methodology is developed: phase and gain control policies incorporate necessary weighting functions and determine them in a rational and systematic way; on the other hand, with the appropriate 
weighting functions efficient $H_{\infty}$ control algorithms can automatically realize phase and gain control policies and generate a satisfactory $H_{\infty}$ controller. The proposed control methodology makes full use of phase and gain control policies and the $H_{\infty}$ control. It can be used for both SISO and MIMO systems with collocated or non-collocated sensors and actuators. In this article, this control methodology is validated on a non-collocated SISO piezoelectric cantilever beam. Both numerical simulations and experimental results demonstrate the effectiveness of the proposed control methodology.

Since the proposed control methodology is general and systematic, it can be applied to more complicated and practical structures, e.g. the suspension systems (Zhong et al., 2010) where several sensors and actuators can be used. To quantitatively verify the robustness properties of the closed-loop system with the designed $H_{\infty}$ controller, deterministic and probabilistic robustness analysis can be employed (Zhang et al., 2013). In the following research, with the finite dimensional LMI optimization (Dinh et al., 2005) the proposed control methodology can be extended to linear parameter-varying and nonlinear systems.

\section{References}

Aoki, Y., Gardonio, P., Elliott, S., 2008. Modelling of a piezoceramic patch actuator for velocity feedback control. Smart Materials and Structures 17, 015052.

Aphale, S., Fleming, A., Moheimani, S., 2007. Integral resonant control of collocated smart structures. Smart Materials and Structures 16 (2), 439. 
Bai, Y., Grigoriadis, K., 2005. $H_{\infty}$ collocated control of structural systems: An analytical bound approach. Journal of Guidance, Control, and Dynamics $28,850-855$.

Balas, M., 1979. Direct velocity feedback control of large space structures. Journal of Guidance, Control, and Dynamics 2 (3), 253-253.

Barrault, G., Halim, D., Hansen, C., 2007. High frequency spatial vibration control using $H_{\infty}$ method. Mechanical Systems and Signal processing 21 (4), 1541-1560.

Barrault, G., Halim, D., Hansen, C., Lenzi, A., 2008. High frequency spatial vibration control for complex structures. Applied Acoustics 69 (11), 933944.

Bayon de Noyer, M., Hanagud, S., 1998a. A comparison of $H_{2}$ optimized design and cross-over point design for acceleration feedback control. In: 39th AIAA/ASME/ASCE/AHS, Structures, Structural Dynamics and Materials Conference. pp. 3250-3258.

Bayon de Noyer, M., Hanagud, S., 1998b. Single actuator and multi-mode acceleration feedback control. Journal of Intelligent Material Systems and Structures 9 (7), 522-533.

Bendat, J., Piersol, A., 1980. Engineering Applications of Correlation and Spectral Analysis. John Wiley and Sons.

Bhattacharya, P., Suhail, H., Sinha, P., 2002. Finite element analysis and distributed control of laminated composite shells using LQR/IMSC approach. Aerospace Science and Technology 6 (4), 273-281. 
Bhikkaji, B., Moheimaniand, S., Petersen, I., 2012. A negative imaginary approach to modeling and control of a collocated structure. IEEE/ASME Transactions on Mechatronics 17 (4), 717-727.

Blondel, D., Tsitsiklis, J., 2000. A survey of computational complexity results in systems and control. Automatica 36 (9), 1249-1274.

Bourlès, H., Kwan, G., 2010. Linear Systems. John Wiley and Sons.

Braatz, P., Young, P., Doyle, J., Morari, M., 1994. Computational complexity of $\mu$ calculation. IEEE Transactions on Automatic Control 39 (5), 10001002.

Caracciolo, R., Richiedei, D., Trevisani, A., Zanotto, V., 2005. Robust mixednorm position and vibration control of flexible link mechanisms. Mechatronics 15 (7), 767-791.

Carrere, F., Duc, G., Font, S., 1997. Commande fréquentielle robusteapplication aux paliers magnétiques. Techniques de l'ingénieur. Informatique industrielle 2 (R7432), R7432-1.

Chang, W., Gopinathan, S., Varadan, V., Varadan, V., 2002. Design of robust vibration controller for a smart panel using finite element model. Journal of Vibration and Acoustics 124, 265-276.

Chen, Y., Zhang, W., Gao, H., 2010. Finite frequency $H_{\infty}$ control for building under earthquake excitation. Mechatronics 20 (1), 128-142.

Crassidis, J., Baz, A., Wereley, N., 2000. $H_{\infty}$ control of active constrained layer damping. Journal of Vibration and Control 6 (1), 113. 
Demetriou, A., Grigoriadis, K., Sweeney, R., 2009. Collocated $H_{\infty}$ control of a cantilevered beam using an analytical upper-bound approach. Journal of Intelligent Material Systems and Structures 20 (7), 865-873.

Desoer, C., Vidyasagar, M., 1975. Feedback Systems: Input-Output Properties. New York: Academic Press.

Dinh, M., Scorletti, G., Fromion, V., Magarotto, E., 2005. Parameter dependent $H_{\infty}$ control by finite dimensional LMI optimization: application to trade-off dependent control. International Journal of Robust and Nonlinear Control 15 (9), 383-406.

Douat, L., Queinnec, I., Garcia, G., Michelin, M., Pierrot, F., 2011. $H_{\infty}$ control applied to the vibration minimization of the parallel robot par2. In: IEEE International Conference on Control Applications. pp. 947-952.

Doyle, J., 1978. Guaranteed margins for LQG regulators. IEEE Transactions on Automatic Control 23 (4), 756-757.

Doyle, J., 1982. Analysis of feedback systems with structured uncertainties. In: IEE Proceedings D on Control Theory and Applications. pp. 242-250.

Doyle, J., 1985. Structured uncertainty in control system design. In: 24th IEEE Conference on Decision and Control. pp. 260-265.

Doyle, J., Glover, K., Khargonekar, P., Francis, B., 1989. State-space solutions to standard $H_{2}$ and $H_{\infty}$ control problems. IEEE Transactions on Automatic Control 34 (8), 831-847. 
Doyle, J., Packard, A., Zhou, K., 1991. Review of LFTs, LMI's, and $\mu$. In: 30th IEEE Conference on Decision and Control. pp. 1227-1260.

Fan, M., Tits, A., Doyle, J., 1991. Robustness in the presence of mixed parametric uncertainty and unmodeled dynamics. IEEE Transactions on Automatic Control 36 (1), 25-38.

Filardi, G., Sename, O., Besançon-Voda, A., Schröder, H., 2003. Robust $H_{\infty}$ control of a DVD drive under parametric uncertainties. In: 17th European Control Conference. pp. 1-7.

Font, S., Duc, G., Carrere, F., 1994. $H_{\infty}$ control of a magnetic bearing. In: 3rd IEEE Conference on Control Applications. Vol. 1. pp. 581-585.

Forrai, A., Hashimoto, S., Isojima, A., Funato, H., Kamiyama, K., 2001. Gray box identification of flexible structures: application to robust active vibration suppression control. Earthquake Engineering \& Structural Dynamics 30 (8), 1203-1220.

Freudenberg, J., Morton, B., 1992. Robust control of a booster vehicle using $H_{\infty}$ and SSV techniques. In: 31st IEEE Conference on Decision and Control. pp. 2448-2453.

Gahinet, P., Apkarian, P., 1994. A linear matrix inequality approach to $H_{\infty}$ control. International Journal of Robust and Nonlinear Control 4, 421-448.

Goh, C., Yan, W., 1996. Approximate pole placement for acceleration feedback control of flexible structures. Journal of Guidance, Control, and Dynamics 19 (1), 256-259. 
Griggs, W., Anderson, B., Lanzon, A., 2007. A "mixed" small gain and passivity theorem in the frequency domain. Systems \& Control Letters 56, 596-602.

Gu, D., Petkov, P., Konstantinov, M., 2005. Robust Control Design with MATLAB. Springer Verlag, London.

Hong, S., Park, C., Park, H., 2006. Vibration control of beams using multiobjective state-feedback control. Smart Materials and Structures 15, 157.

Huo, L., Song, G., Li, H., Grigoriadis, K., 2008. $H_{\infty}$ robust control design of active structural vibration suppression using an active mass damper. Smart Materials and Structures 17, 015021.

Iorga, L., Baruh, H., Ursu, I., 2008. A review of $H_{\infty}$ robust control of piezoelectric smart structures. Applied Mechanics Reviews 61 (4), 040802.

Iorga, L., Baruh, H., Ursu, I., 2009. $H_{\infty}$ control with $\mu$-analysis of a piezoelectric actuated plate. Journal of Vibration and Control 15 (8), 1143-1171.

Jabbari, F., Schmitendorf, W., Yang, J., 1995. $H_{\infty}$ control for seismic-excited buildings with acceleration feedback. Journal of Engineering Mechanics 121 (9), 994-1002.

Jemai, B., Ichchou, M., Jézéquel, L., Noe, M., 1999. An assembled plate active control damping set-up: optimization and control. Journal of Sound and Vibration 225 (2), 327-343.

Kar, I., Miyakura, T., Seto, K., May 2000a. Bending and torsional vibration 
control of a flexible plate structure using $H_{\infty}$-based robust control law. IEEE Transactions on Control Systems Technology 8 (3), 545 -553.

Kar, I., Seto, K., Doi, F., 2000b. Multimode vibration control of a flexible structure using $H_{\infty}$-based robust control. IEEE/ASME Transactions on Mechatronics 5 (1), 23-31.

Khalil, H., 1996. Nonlinear Systems, 2nd Edition. Prentice-Hall New Jersey.

Khot, S., Yelve, N., Tomar, R., Desai, S., Vittal, S., 2011. Active vibration control of cantilever beam by using PID based output feedback controller. Journal of Vibration and Control 18 (3), 366-372.

Kim, S., Oh, J., 2013. A modal filter approach to non-collocated vibration control of structures. Journal of Sound and Vibration 332 (9), 2207-2221.

Lanzon, A., Petersen, I., 2008. Stability robustness of a feedback interconnection of systems with negative imaginary frequency response. IEEE Transactions on Automatic Control 53 (4), 1042-1046.

Li, P., Cheng, L., Li, Y., Chen, N., 2003. Robust control of a vibrating plate using $\mu$-synthesis approach. Thin-Walled Structures 41 (11), 973-986.

Liu, T., Hua, H., Zhang, Z., 2004. Robust control of plate vibration via active constrained layer damping. Thin-Walled Structures 42 (3), 427-448.

Ma, K., Ghasemi-Nejhad, M., 2005. Adaptive simultaneous precision positioning and vibration control of intelligent composite structures. Journal of Intelligent Material Systems and Structures 16 (2), 163-174. 
Meirovitch, L., 1986. Elements of Vibration Analysis, 2nd Edition. Sydney McGraw Hill.

Moheimani, S., Fleming, A., 2006. Piezoelectric Transducers for Vibration Control and Damping. Springer-Verlag, London.

Morris, J., Apkarian, P., Doyle, J., 1992. Synthesizing robust mode shapes with $\mu$ and implicit model following. In: 1st IEEE Conference on Control Applications. Vol. 2. pp. 1018-1023.

Piefort, V., 2001. Finite element modelling of piezoelectric active structures. Ph.D. thesis, Universite Libre de Bruxelles.

Pota, H., Moheimani, S., Smith, M., 1999. Resonant controllers for flexible structures. In: 38th IEEE Conference on Decision and Control. Vol. 1. pp. $631-636$.

Pota, H., Moheimani, S., Smith, M., 2002. Resonant controllers for smart structures. Smart Materials and Structures 11, 1-8.

Qiu, Z., Han, J., Zhang, X., Wang, Y., Wu, Z., 2009. Active vibration control of a flexible beam using a non-collocated acceleration sensor and piezoelectric patch actuator. Journal of Sound and Vibration 326 (3), 438-455.

Qiu, Z., Zhang, X., Wu, H., Zhang, H., 2007. Optimal placement and active vibration control for piezoelectric smart flexible cantilever plate. Journal of Sound and Vibration 301 (3), 521-543.

Qu, W., Sun, J., Qiu, Y., 2004. Active control of vibration using a fuzzy control method. Journal of Sound and Vibration 275 (3), 917-930. 
Rao, A., Natesan, K., Bhat, M., Ganguli, R., 2007. Experimental demonstration of $H_{\infty}$ control based active vibration suppression in composite fin-tip of aircraft using optimally placed piezoelectric patch actuators. Journal of Intelligent Material Systems and Structures 19 (6), 651-669.

Rohrs, C., Valavani, L., Athans, M., Stein, G., 1985. Robustness of continuous-time adaptive control algorithms in the presence of unmodeled dynamics. IEEE Transactions on Automatic Control 30 (9), 881-889.

Sadri, A., Wynne, R., Wright, J., 1999. Robust strategies for active vibration control of plate-like structures: theory and experiment. Proceedings of the Institution of Mechanical Engineers, Part I: Journal of Systems and Control Engineering 213 (6), 489-504.

Schoukens, J., Pintelon, R., 1991. Identification of Linear Systems, A Pratical Guide to Accurate Modeling. Pergamon Press, New York.

Scorletti, G., Fromion, V., 2009. Automatique fréquentielle avancée. Ecole Centrale de Lyon, Master EEAP GSA, Université de Lyon 1, INSA de Lyon,http://cel.archives-ouvertes.fr/cel-0042384.

Sefton, J., Glover, K., 1990. Pole/zero cancellations in the general $H_{\infty}$ problem with reference to a two block design. Systems \& Control Letters 14 (4), 295-306.

Seto, K., Kar, I., 2000. A comparative study on $H_{\infty}$ based vibration controller of a flexible structure system. In: 2000 American Control Conference. pp. $513-518$. 
Sim, E., Lee, S., 1993. Active vibration control of flexible structures with acceleration feedback. Journal of Guidance, Control, and Dynamics 16 (2), $413-415$.

Skogestad, S., Postlethwaite, I., 2005. Multivariable Feedback ControlAnalysis and Design. John Wiley and Sons.

Song, Z., Lanzon, A., Patra, S., Petersen, I., 2012. Robust performance analysis for uncertain negative-imaginary systems. International Journal of Robust and Nonlinear Control 22 (3), 262-281.

Stalford, H., 1987. Robust control of uncertain systems in the absence of matching conditions: Scalar input. In: 26th IEEE Conference on Decision and Control. Vol. 26. pp. $1298-1307$.

Wang, S., 2003. Robust active control for uncertain structural systems with acceleration sensors. Journal of Structural Control 10 (1), 59-76.

Wang, S., Yeh, H., Roschke, P., 2001. Robust control for structural systems with parametric and unstructured uncertainties. Journal of Vibration and Control 7 (5), 753-772.

Xie, S., Zhang, X., Zhang, J., Yu, L., 2004. $H_{\infty}$ robust vibration control of a thin plate covered with a controllable constrained damping layer. Journal of Vibration and Control 10 (1), 115-133.

Yaman, Y., Çalışkan, T., Nalbantoğlu, V., Prasad, E., Waechter, D., 2001. Active vibration control of a smart beam. In: Canada-US CanSmart Workshop on Smart Materials and Structures. pp. 137-147. 
Yaman, Y., Çalışkan, T., Nalbantoğlu, V., Prasad, E., Waechter, D., 2002. Active vibration control of a smart plate. ICAS 2002, International Council of the Aeronautical Sciences, Toronto, Canada,.

Young, P., Dolye, J., 1990. Computation of $\mu$ with real and complex uncertainties. In: 29th IEEE Conference on Decirion and Control. pp. 12301235.

Young, P., Newlin, M., Doyle, J., 1992. Practical computation of the mixed $\mu$ problem. In: 1992 American Control Conference. pp. 2190-2194.

Zhang, K., Scorletti, G., Ichchou, M., Mieyeville, F., 2013. Robust active vibration control of piezoelectric flexible structures using deterministic and probabilistic analysis, submitted to Journal of Intelligent Material Systems and Structures.

Zhang, X., Shao, C., Li, S., Xu, D., Erdman, A., 2001. Robust $H_{\infty}$ vibration control for flexible linkage mechanism systems with piezoelectric sensors and actuators. Journal of Sound and Vibration 243 (1), 145-155.

Zhong, X., Ichchou, M., Gillot, F., Saidi, A., 2010. A dynamic-reliable multiple model adaptive controller for active vehicle suspension under uncertainties. Smart Materials and Structures 19 (045007). 